\title{
TLR4 activation mediates kidney ischemia/reperfusion injury
}

\author{
Huiling Wu, ${ }^{1}$ Gang Chen, ${ }^{1}$ Kate R. Wyburn, ${ }^{1}$ Jianlin Yin, ${ }^{1}$ Patrick Bertolino,, ${ }^{1}$ Josette M. Eris, ${ }^{1}$ \\ Stephen I. Alexander, ${ }^{2}$ Alexandra F. Sharland, ${ }^{1}$ and Steven J. Chadban ${ }^{1}$ \\ ${ }^{1}$ Collaborative Transplant Research Group, Royal Prince Alfred Hospital and Bosch Institute, Faculty of Medicine, University of Sydney, Sydney, \\ New South Wales, Australia. ${ }^{2}$ Centre for Kidney Research, Children's Hospital at Westmead, Sydney, New South Wales, Australia.
}

\begin{abstract}
Ischemia/reperfusion injury (IRI) may activate innate immunity through the engagement of TLRs by endogenous ligands. TLR4 expressed within the kidney is a potential mediator of innate activation and inflammation. Using a mouse model of kidney IRI, we demonstrated a significant increase in TLR4 expression by tubular epithelial cells (TECs) and infiltrating leukocytes within the kidney following ischemia. TLR4 signaling through the MyD88-dependent pathway was required for the full development of kidney IRI, as both $T L R 4^{-/-}$and

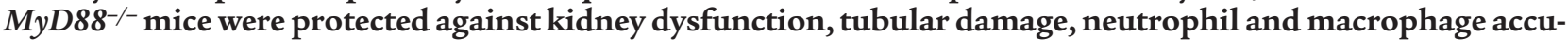
mulation, and expression of proinflammatory cytokines and chemokines. In vitro, WT kidney TECs produced proinflammatory cytokines and chemokines and underwent apoptosis after ischemia. These effects were attenuated in $\mathrm{TLR4}^{-/-}$and $\mathrm{MyD88^{-/- }}$ TECs. In addition, we demonstrated upregulation of the endogenous ligands high-mobility group box 1 (HMGB1), hyaluronan, and biglycan, providing circumstantial evidence that one or more of these ligands may be the source of TLR4 activation. To determine the relative contribution of TLR4 expression by parenchymal cells or leukocytes to kidney damage during IRI, we generated chimeric mice. $T L R 4^{-/-}$mice engrafted with WT hematopoietic cells had significantly lower serum creatinine and less tubular damage than WT mice reconstituted with $T L R 4^{-/-} \mathrm{BM}$, suggesting that TLR4 signaling in intrinsic kidney cells plays the dominant role in mediating kidney damage.
\end{abstract}

\section{Introduction}

Ischemia/reperfusion injury (IRI) is an inevitable consequence of the procedure of kidney transplantation and has a negative impact on both short- and long-term graft survival (1-3). The initial nonimmune injury leads to the activation of an innate immune response causing variable degrees of tissue damage (4-8). Modulation of the graft microenvironment by this early innate response may be a prerequisite for the full development of adaptive alloimmunity and subsequent allograft rejection, suggesting a significant interplay between innate and adaptive responses following transplantation $(9,10)$. The mechanisms by which innate immunity is triggered in kidney IRI remain to be fully defined.

TLRs are germline-encoded pattern-recognition receptors that are highly conserved in species as diverse as Drosophila and humans (11). These receptors recognize molecular motifs shared by large groups of microorganisms, such as LPS, flagellin, and CPG DNA $(12-14)$. TLRs are expressed by cells of the immune system, such as macrophages, DCs, neutrophils, B cells, and NK cells. TLRs may also be expressed in tissue by cell types, including kidney tubular epithelial cells (TECs) and mesangial cells in response to injury (15). Signaling via TLRs is dependent upon association with a group of cytoplasmic adaptor molecules, principally MyD88 and Toll/IL-1 receptor domain-containing adaptor inducing IFN- $\beta$ (TRIF) (16). Downstream effects of TLR engagement vary, depending upon the cell type in which the receptors are expressed. They

Nonstandard abbreviations used: HAS, hyaluronan synthase; HMGB1, high-mobility group box 1; HPF, high-power field; IP10, IFN-inducible protein 10; IRI, ischemia/ reperfusion injury; MCP-1, monocyte chemoattractant protein-1; MIP-2, macrophage inflammatory protein-2; TEC, tubular epithelial cell; TRIF, Toll/IL-1 receptor domain-containing adaptor inducing IFN- $\beta$.

Conflict of interest: The authors have declared that no conflict of interest exists. Citation for this article: J. Clin. Invest. 117:2847-2859 (2007). doi:10.1172/JCI31008. include the production of proinflammatory cytokines, chemokines, and other soluble mediators that contribute to local inflammation and leukocyte accumulation as well as alterations in the chemokine receptor profile and upregulation of the surface expression of MHC and costimulatory molecules on antigen-presenting cells, which promote an effective adaptive immune response (15, 17). In the context of sepsis, kidney expression of TLR 4 is critical in mediating LPS-induced acute kidney failure via proinflammatory cytokine release and subsequent kidney damage (18).

Endogenous ligands released from damaged/stressed tissues can also signal through TLRs $(19,20)$. These ligands include heat-shock proteins (binding to TLR2 and -4), the nonhistone chromatin-binding protein high-mobility group box 1 (HMGB1) (TLR2 and -4), and ECM components such as hyaluronan (TLR2 and -4), fibronectin (TLR4), heparan sulfate (TLR4), and biglycan (TLR2 and -4) (15, 19, 21-24). Increasing experimental evidence indicates that engagement of the TLRs by such endogenous ligands may be a major trigger of inflammation in response to ischemia. Ischemic hepatocytes upregulate HMGB1, and administration of neutralizing anti-HMGB1 antibody protects against lethal hepatic IRI, whereas administration of recombinant HMGB1 worsens liver injury (25). Activation of the innate immune system by HMGB1 in this model required TLR4dependent signaling (25), consistent with previous observations that $T L R 4^{-/}$mice were protected against hepatic IRI $(26,27)$. TLR4 signaling has also been implicated in the pathogenesis of murine myocardial IRI (28). Ischemic rodent kidney cells, particularly TECs, upregulate expression of mRNA for both TLR2 and TLR4 $(29,30)$, and TLR2 has been found to play an important role in the initiation of inflammatory responses during kidney IRI (31). However, the role of TLR4 in kidney IRI remains to be elucidated. In this study, we tested the hypothesis that endogenous ligands released in the course of kidney IRI can activate innate immunity via TLR4 signaling. 

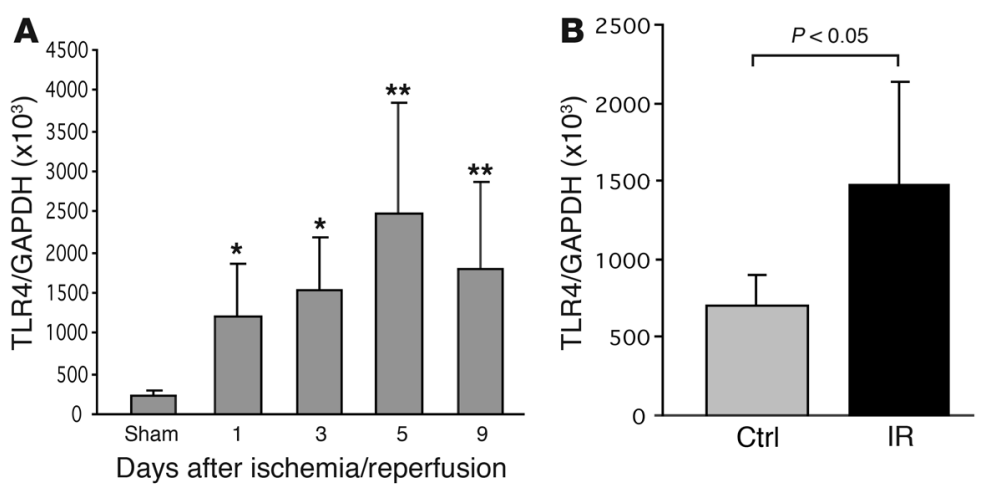

\section{Figure 1}

TLR4 mRNA expression is increased following ischemia/ reperfusion. (A) Ischemia-induced upregulation of TLR4 mRNA expression in the kidney $(n=6-8)$ from day 1 to day 9 after IRI compared with sham-operated controls. $n=6-8$. (B) TECs submitted to ischemia expressed significantly higher levels of mRNA for TLR4 than controls. $P<0.05$. mRNA expression was measured by real-time PCR. The results have been normalized by expressing the number of transcript copies as a ratio to GAPDH. Data are mean \pm SD. Ctrl, control; IR, ischemia/reperfusion. ${ }^{*} P<0.05$; ${ }^{\star *} P<0.01$.

\section{Results}

TLR4 expression is increased in the kidney following kidney ischemia. To determine whether kidney ischemia stimulates TLR4 upregulation, we measured mRNA expression of TLR4 in IRI kidney by real-time PCR. Normal kidney tissue expressed TLR4 at a basal level. TLR4 mRNA levels were significantly increased at days 1 and $3(P<0.05)$ after ischemia, with further upregulation through days 5 and $9(P<0.01$; Figure $1 \mathrm{~A})$. Isolated TECs submitted to ischemia expressed significantly higher mRNA levels of TLR4 than the controls $(P<0.05$; Figure 1B).

Immunostaining demonstrated that TLR 4 protein was expressed by TECs and intrakidney leukocytes at day 1 after IRI and thereafter predominantly by TECs (Figure 2, A and C). Shamoperated controls had very faint staining in occasional tubules only. TLR $4^{+}$leukocytes on day 1 after IRI may have represented resident kidney DCs/macrophages, infiltrating cells, or a com-

\section{Figure 2}

TLR protein expression is increased in the kidney following ischemia/reperfusion. (A) Immunostaining demonstrated that TLR4 protein was expressed by infiltrating cells and TECs at day 1 and then predominantly expressed by TECs on days 3, 5, and 9 after IRI. Original magnification, $\times 200$. (B) TLR4 was expressed by intrarenal leukocyte cells at day 1 after IRI. Magnification, $\times 600$. (C) TLR4 was expressed by tubular cells at day 5 after IRI. Original magnification, $\times 600$. (D) The number of cells expressing TLR4 dramatically increased on day 1 , declining rapidly thereafter. These cells could be resident renal DCs/macrophages or infiltrating leukocytes. (E) Expression levels of TLR4 protein in tubules were significantly increased from day 1 to day 9 after IRI compared with shamoperated controls. ${ }^{* *} P<0.01 ;{ }^{* *} P<0.001$. Data are mean \pm SD. $n=6-8$ per group.
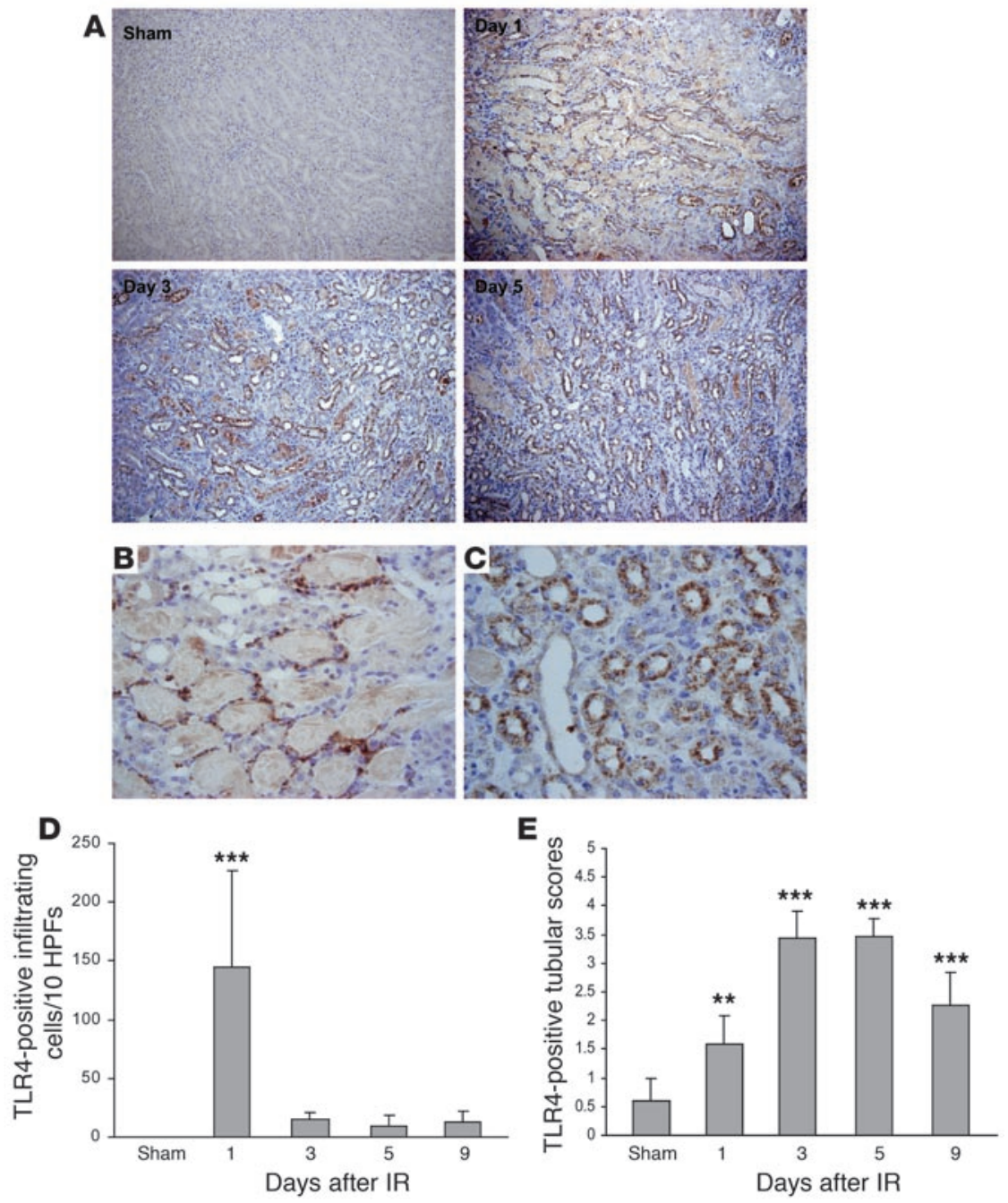


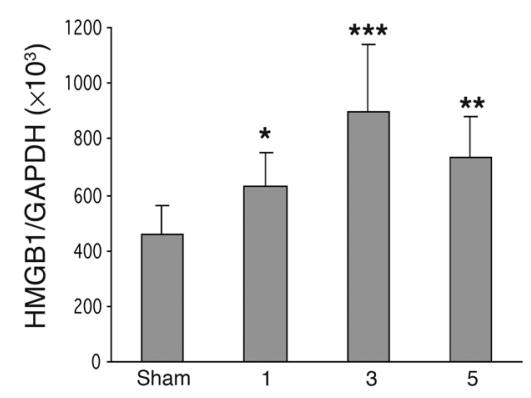

Days after ischemia/reperfusion

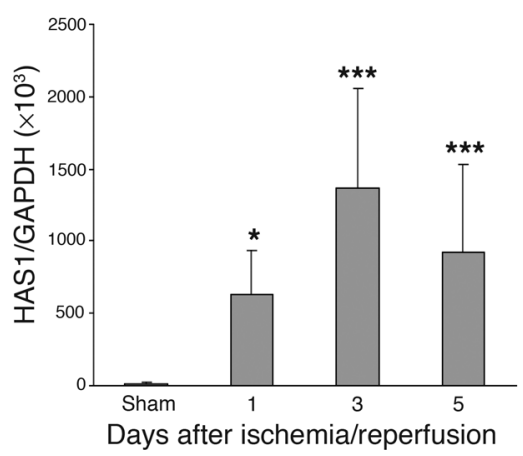

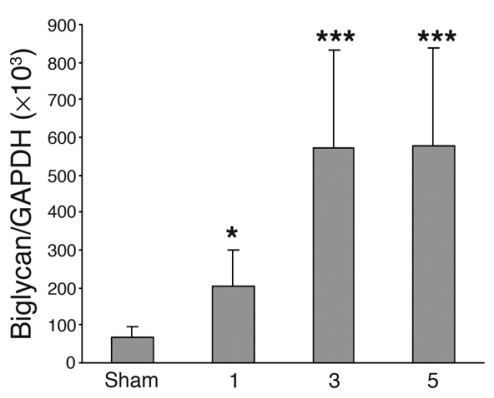

Days after ischemia/reperfusion

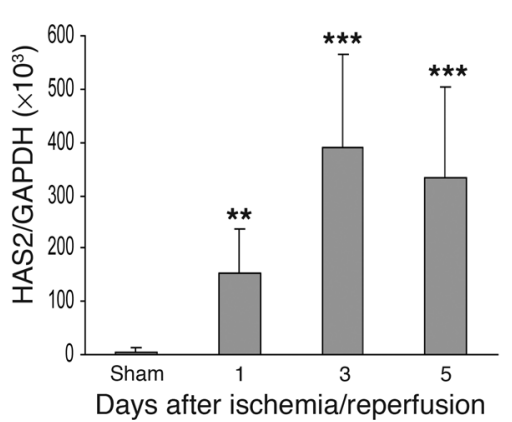

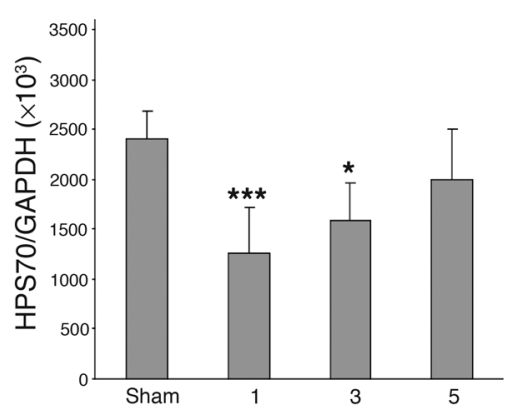

Days after ischemia/reperfusion

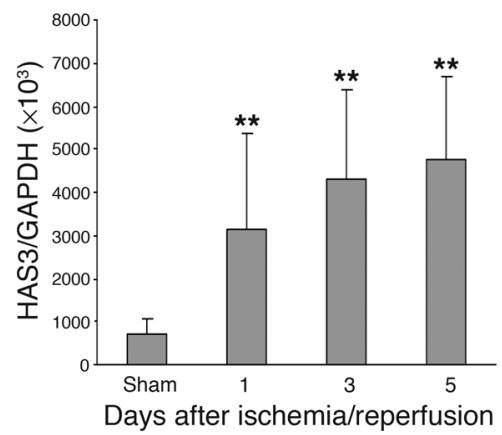

Figure 3

mRNA expression of endogenous ligands for TLR4 expressed in the IRI kidney by real-time PCR. mRNA levels for HMGB1, biglycan, and HAS1, -2, and -3 were significantly increased from day 1 to day 5 after IRI, but HSP70 mRNA levels were not increased. Data shown are mean \pm SD. $n=7-10$ per group. ${ }^{*} P<0.05,{ }^{* \star} P<0.01,{ }^{* * *} P<0.001$.

bination of these (Figure 2B). The number of TLR4+ leukocytes diminished through days 3-5 (Figure 2D). Expression of TLR4 protein by the tubules was most evident in the injured proximal tubules of the medullary ray and the outer medullary strip (Figure 2, A and C). Expression of TLR4 protein in tubules was significantly increased from day 1 to day 9 after IRI compared with that in sham-operated controls (Figure 2E).

Endogenous ligands for TLR4 are expressed in the kidney during IRI. To determine whether ischemia induced upregulation of endogenous TLR ligands in our model, we measured mRNA expression for $H M G B 1$, biglycan, all 3 isoforms of hyaluronan synthase (HAS) reflecting hyaluronan biosynthesis, and HSP70 in IRI kidney by real-time PCR. Levels of mRNA for HMGB1 and biglycan were significantly increased at day 1 after IRI, with further upregulation through days 3 and 5 compared with sham-operated controls (Figure 3). Sham-operated kidneys expressed negligible levels of $H A S$, whereas HAS1, -2, and -3 mRNA expression increased dramatically in IRI kidney from day 1 to day 5 (26- to 136 -fold increase for HAS1; 23- to 68-fold increase for HAS2; 3- to 8-fold increase for $H A S 3$ ) (Figure 3). In contrast, mRNA expression for the inducible form of HSP70 was not increased in IRI kidney on days 1 through 5 compared with sham-operated kidneys (Figure 3).

Consistent with the real-time PCR data, staining with biotinylated hyaluronan-binding protein (b-HABP) showed that IRI induced a progressive increase in interstitial hyaluronan expression in the medullary ray and the outer medullary strip from day 1 to day 9 (Figure 4A). There was minimal expression in sham-operated kidney. Morphometric analysis of hyaluronan expression showed that both the area and intensity of hyaluronan staining increased from day 1 to day 9 after IRI compared with sham-operated controls
(Figure 4B). Immunofluorescent staining demonstrated expression of HMGB1 by TECs on days 1 through 5 after IRI with negligible expression in sham-operated controls (Figure 4C). Western blot showed that protein expression of HSP70 was not significantly increased between IRI and sham-operated kidneys (Figure 4D).

$T L R 4^{-/}$mice are protected against kidney IRI. To determine whether the full development of kidney IRI was dependent on TLR4 signaling, we used $T L R 4^{-/}$mice in the ischemia/reperfusion model. TLR4 $4^{--}$mice were protected against the effects of ischemia, exhibiting significantly lower serum creatinine and less tubular damage than WT controls. As shown in Figure 5, IRI caused kidney dysfunction in WT mice with a peak serum creatinine of $109 \pm 39.1 \mu \mathrm{mol} / 1$ at day 1 after ischemia/reperfusion, which gradually fell thereafter but remained elevated $(27.2 \pm 4.9 \mu \mathrm{mol} / \mathrm{l})$ at day 9 after IRI as compared with sham-operated mice $(14.8 \pm 3.8 \mu \mathrm{mol} / \mathrm{l})$. In contrast, there was a very modest rise in serum creatinine in TLR4 $4^{-/}$mice $(29 \pm 6.1 \mu \mathrm{mol} / \mathrm{l})$ on day 1 , and creatinine remained lower than in WT controls at all time points thereafter.

The functional data correlated with histological kidney tubular damage. As shown in Figure 6A, in WT mice there was severe tubular damage, as evidenced by widespread tubular necrosis, loss of the brush border, cast formation, and tubular dilatation at the corticomedullary junction, maximal at day 1 with gradual recovery by day 9, whereas $T L R 4^{-/-}$mice showed significantly less tubular damage as compared with WT controls from day 1 to day 5 after IRI (Figure 6B). Sham-operated mice incurred no tubular injury.

The adaptor protein MyD88 is important in TLR4-mediated IRI. The major signaling pathway for TLRs proceeds via an adaptor protein, MyD88. To determine whether the MyD88 signaling pathway is involved, we also used $M y D 88^{-/-}$mice in this ischemia/reperfusion 

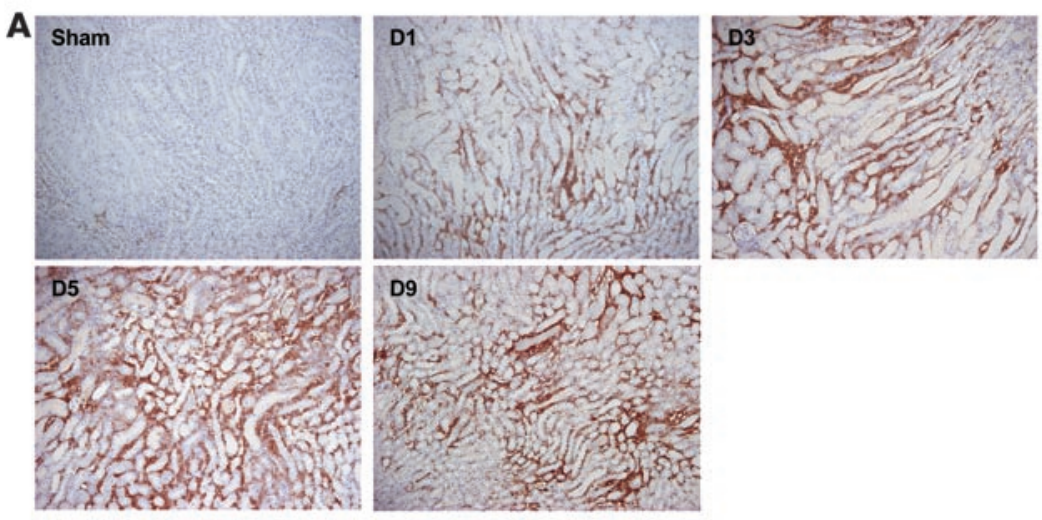

B

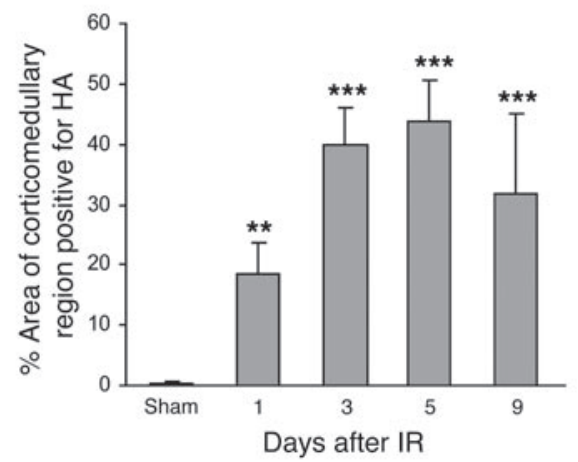

C
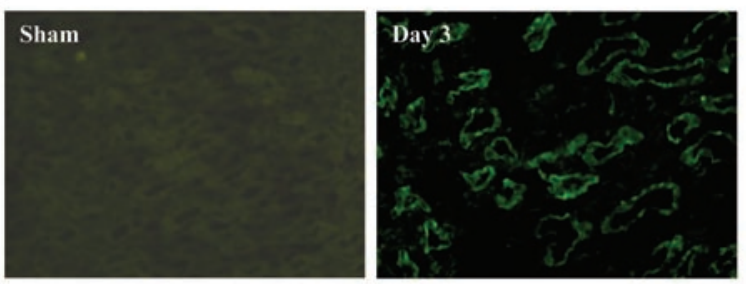

D

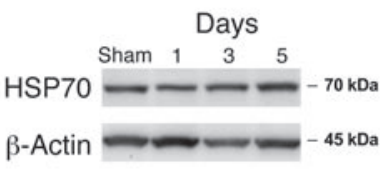

\section{Figure 4}

IRI induces a marked and progressive increase in interstitial hyaluronan expression in WT mice. Representative sections of the kidney are stained for hyaluronan (HA) using biotinylated hyaluronan-binding protein $(b-H A B P)$ (original magnification, $\times 200)$ from day 1 to day 9 after IRI (A). (B) Analysis of $\mathrm{HA}$ expression showed that the area of HA-positive staining was significantly increased in the renal interstitium from day 1 to day 9 after IRI compared with shamoperated controls. The comparison between IRI kidney from day 1 to day 9 and shamoperated control is indicated by asterisks. ${ }^{* *} P<0.01 ;{ }^{* * *} P<0.001$. (C) Immunofluorescent staining indicates upregulation of HMGB1 expression by TECs at day 3 after IRI compared with sham-operated controls. Original magnification, $\times 400$. (D) Western blot showed that protein expression of HSP70 was not significantly increased in IRI versus sham-operated kidneys. model. $M y D 88^{-/-}$mice were also protected against kidney IRI with significantly lower serum creatinine versus WT controls from day 1 to day 9 as shown in Figure 5 and also manifested less tubular damage than WT controls as shown in Figure 6.

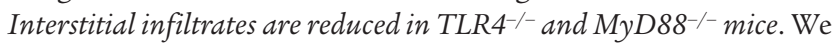
further analyzed the cellular infiltrates in IRI kidney. Prominent interstitial neutrophil infiltration was observed in WT kidney at day 1 after ischemia/reperfusion; this largely subsided by day 9 (Figure 7). Neutrophil infiltration was significantly less in
TLR4 $4^{-/-}$and $M y D 88^{-/-}$mice than in WT controls from day 1 to day 5 (Figure 7B).

Interstitial macrophages progressively accumulated in WT animals from day 1 to day 5 after IRI, then declined slightly by day 9 (Figure 8). Macrophage infiltration was most pronounced in the outer medulla at day 1 and day 3 and extended nearly to the cortex at day 5 and day 9. Compared with WT controls, TLR $4^{--}$and $M y D 88^{-/-}$mice had significantly less interstitial macrophages at all time points (Figure 8B).

\section{Figure 5}

TLR4 $4^{-/-}$(black bars) and MyD88-/- (white bars) mice were protected against renal IRI with significantly lower serum creatinine compared with WT controls (gray bars) from day 1 to day 9 after reperfusion. Sham-operated mice had normal serum creatinine $(10-20 \mu \mathrm{mol} / \mathrm{l})$. Data are mean \pm SD. $n=6-8$ per group. The comparison between TLR4 $4^{-/-}$mice or $M y D 88^{-/-}$mice and WT controls is indicated by asterisks. ${ }^{\star} P<0.05 ;{ }^{\star \star} P<0.01 ;{ }^{\#} P<0.001$.

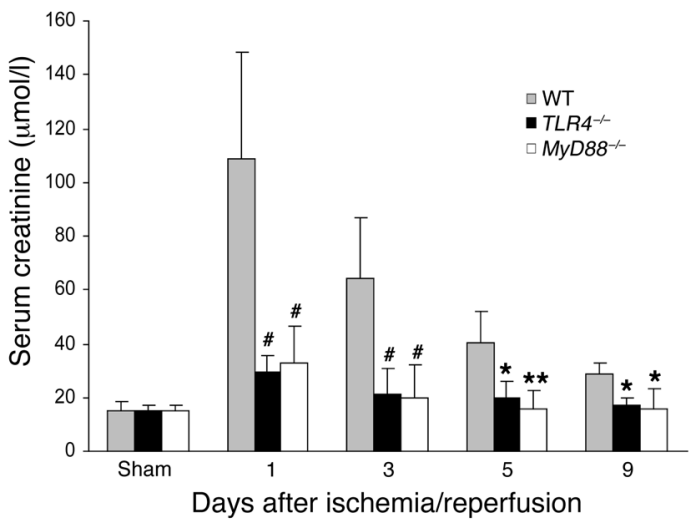

Days after ischemia/reperfusion 

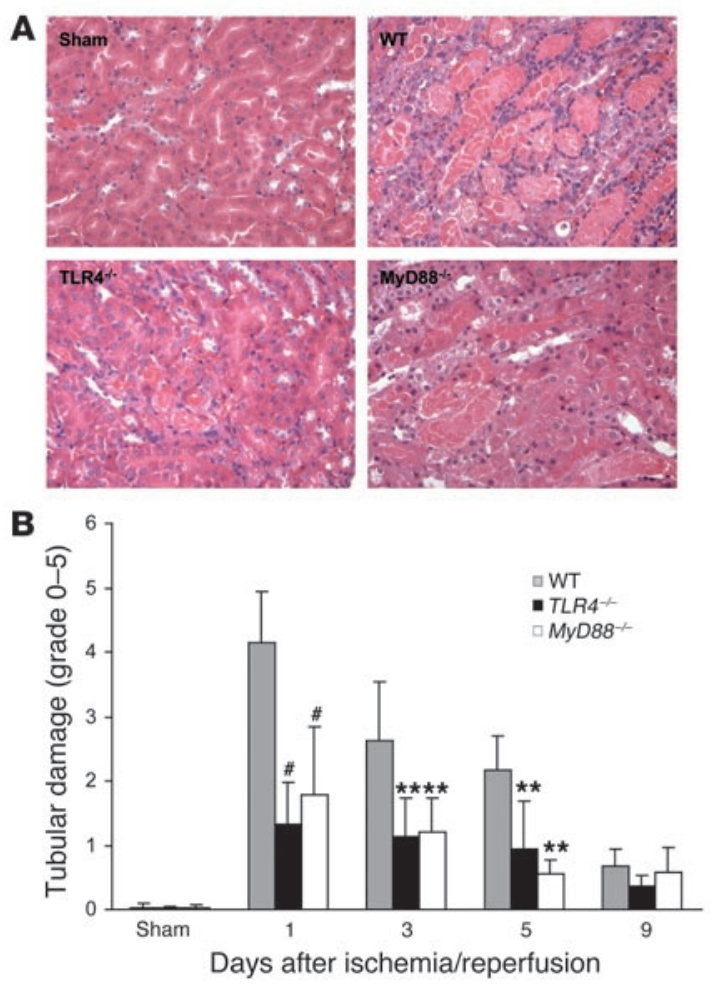

TLR4 mediates proinflammatory cytokine and chemokine expression in the kidney during IRI. To further determine the effects of TLR4 signaling in the IRI kidney model, we examined the expression of known TLR4 downstream cytokines and chemokines. IL- 6 and TNF- $\alpha$ mRNAs were strongly upregulated in WT kidney on days 1-5, peaking on day 3 ( $P<0.001$ compared with sham-operated con-

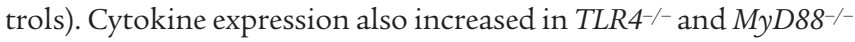
mice but to a much lesser extent $(P<0.05-0.001$; Figure 9). $I L-1 \beta$ mRNA levels in the kidney were significantly increased in WT mice on day 1 following ischemia, and this increase was abrogated in TLR4 $4^{-/-}$and MyD88 $8^{-/-}$kidneys. (Figure 9).

Chemokine (macrophage inflammatory protein-2 [MIP-2] and monocyte chemoattractant protein-1 [MCP-1]) mRNA levels in the kidney increased by several hundred-fold in WT mice after IRI compared with sham-operated controls $(P<0.001)$. Upregulation of chemokine expression was greatly attenuated in $T L R 4^{--}$and $M y D 88^{-/-}$kidneys compared with WT controls at all time points (Figure 9). mRNA levels for the IFN- $\beta$ inducible chemokine gene IFN-inducible protein 10 (IP10) also increased in the kidneys of WT mice after IRI on days $1-5(P<0.05-0.001)$. However, unlike the other chemokines, no significant differences in IP10 mRNA

\section{Figure 7}

Neutrophil accumulation within the interstitium of the kidney was significantly less in $\mathrm{TLR}^{-{ }^{--}}$and $M y D 88^{-/-}$mice versus WT controls from day 1 to day 5 after reperfusion. (A) Representative sections of kidney stained for neutrophils by immunohistochemistry. Original magnification, $\times 200$. (B) Analysis of neutrophil infiltrate in WT (gray bars), TLR4 $4^{-/}$(black bars), and MyD88 ${ }^{-/-}$(white bars) mouse kidney (numbers/10 HPFs). Data shown are mean \pm SD. $n=6-8$ per group. The comparison between TLR4 $4^{--}$mice or $M y D 88^{-1-}$ mice and WT controls is indicated by asterisks. ${ }^{*} P<0.05$; ${ }^{\#} P<0.001$.

\section{Figure 6}

Tubular injury in $T L R 4^{-/}$and $M y D 88^{-/-}$kidney was significantly less than that seen in kidney from WT mice. (A) Representative sections of outer medulla from sham-operated, WT, TLR4 $4^{--}$, and MyD88 -/- mice 1 day after reperfusion (H\&E stained). Original magnification, $\times 200$. (B) Semiquantitative analysis of tubular damage in WT (gray bars), TLR4 $4^{-/-}$(black bars), and MyD88-/- (white bars) mouse kidney from day 1 to day 9 after reperfusion. Data shown are mean \pm SD. $n=6-8$ per group. The comparison between TLR4 $4^{-/-}$mice or $M y D 88^{-/-}$mice and WT controls is indicated by asterisks. ${ }^{* *} P<0.01,{ }^{\#} P<0.001$.

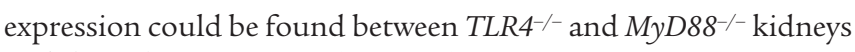
and those from WT mice (Figure 9).

Protein levels of cytokines (IL-6, IL- $1 \beta$, and TNF- $\alpha$ ) and chemokines (MIP-2 and MCP-1) in kidney homogenates from TLR4-/-, $M y D 88^{-/-}$, and WT mice on day 1 to day 5 after IRI, measured by ELISA, generally reflected mRNA expression (Figure 10). IL-6, IL-1 $\beta$, and MCP-1 protein levels in IRI kidney were significantly increased in WT mice compared with sham-operated controls $(P<0.001)$. Limited increases in these protein levels occurred in $T L R 4^{--}$and $M y D 88^{-/-}$mice, and protein levels were always significantly reduced compared with those in WT mice. For MIP-2, protein levels were equivalent in all 3 groups on day 1 but remained elevated in the WT mice while declining rapidly in TLR4 $4^{-/}$and $M y D 88^{-/-}$mice. Low levels of TNF- $\alpha$ protein expression were detected in some IRI kidneys in WT mice but not in $T L R 4^{-/-}$and $M y D 88^{-/-}$kidney homogenates.

Cytokine and chemokine mRNA expression is also decreased in TLR4--and MyD88-1- primary cultured TECs undergoing transient ischemia in vitro. Kidney TECs in mice are known to express TLR1, -2, -3, -4, and -6 (15). Primary mouse kidney TECs in culture secrete CC che-
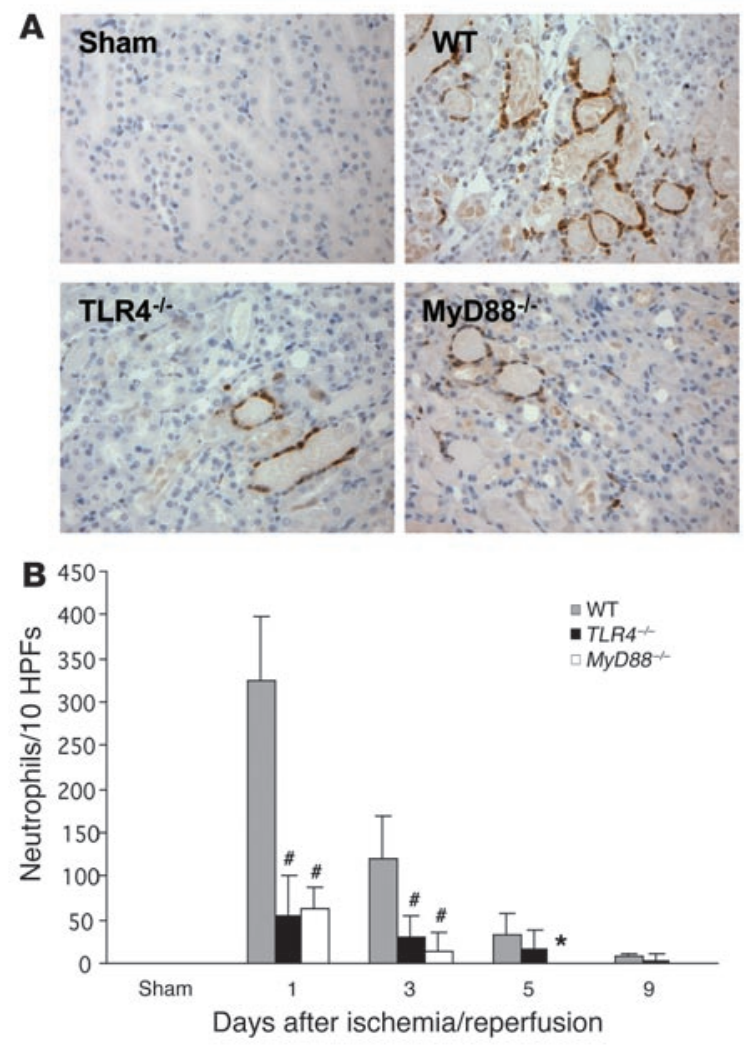
A
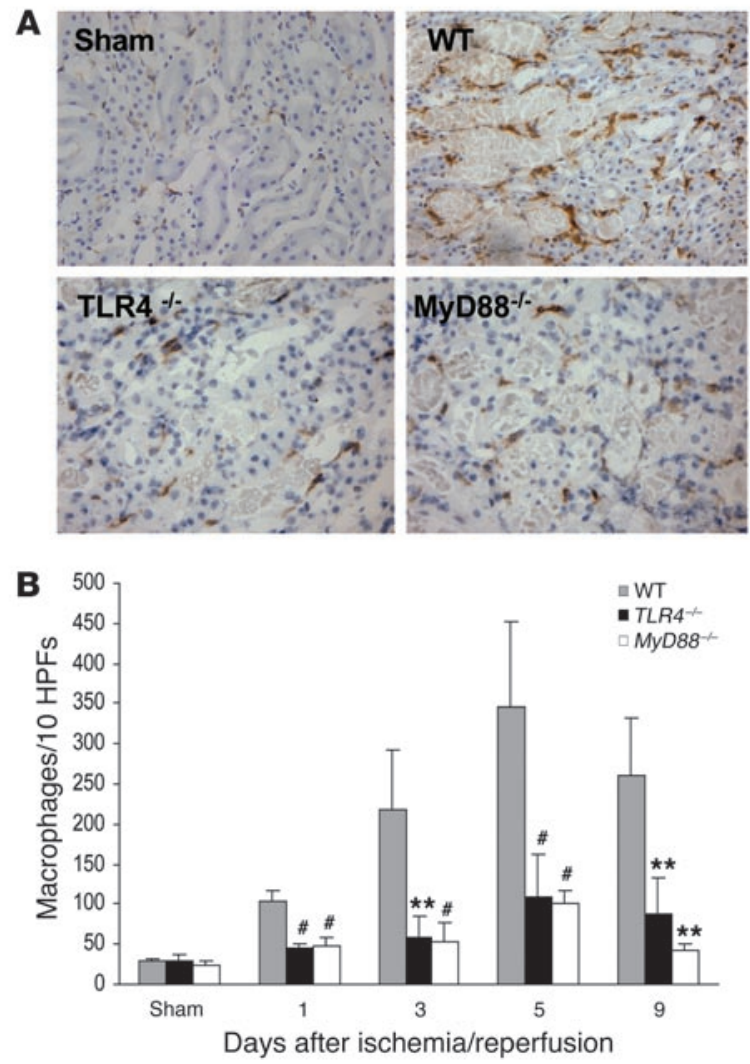

mokines and proinflammatory cytokines in response to stimulation via TLR2 or -4 (32). To examine mRNA expression of cytokines and chemokines by kidney TECs undergoing transient ischemia in vitro, primary cultures of TECs were established. The purity of these cultures was defined by positive staining for the epithelial marker cytokeratin and always exceeded 95\% (range 96\%-100\%; Figure 11). As shown in Figure 11B, WT TECs submitted to ischemia expressed significantly higher mRNA levels of cytokines (IL-6, $I L-1 \beta$, and $T N F-\alpha)$ and chemokines (MIP-2 and MCP-1) as compared with nonischemic controls $(P<0.05)$. TLR $4^{-/-}$and $M y D 88^{-/-}$TECs submitted to ischemia in vitro showed reduced cytokine and chemokine gene expression versus WT controls $(P<0.001)$ and indeed showed expression level equal to nonischemic TEC controls.

Apoptosis is decreased in TLR4-/- and MyD88-1- TECs undergoing tran-

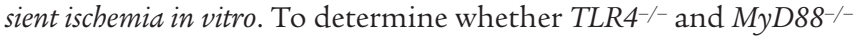
TECs were protected against ischemia-mediated cell death, TECs in primary culture were subjected to transient ischemia, then stained with annexin V to identify apoptotic cells (Figure 12A). Flow cytometric analysis showed that the proportion of apoptotic cells increased from $6.7 \%$ in the nonischemic controls to $18.7 \%$ after ischemia in the WT TECs $(P<0.001)$ (Figure 12B). In con-

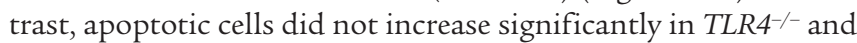
$M y D 88^{-1-}$ TECs after ischemia. TUNEL assay confirmed these findings, with an increase in apoptotic cells seen in the WT though not TLR4 $4^{-/}$or $M y D 88^{-/-}$TECs after ischemia (Figure 12C).

TLR4-mediated kidney IRI requires functional TLR4 signaling on kidney parenchymal cells. As TLR4 was expressed on leukocytes and intrinsic kidney cells, we next determined the relative importance of TLR4 signaling through kidney parenchymal cells or BM-derived cells in the pathogenesis of kidney IRI by generating BM chimeric mice.

\section{Figure 8}

Macrophage accumulation within the interstitium of the kidney was significantly less in $\mathrm{TLR4}^{-/-}$and $M y D 88^{-/-}$mice versus WT controls at all time points $(P<0.05)$. (A) Representative sections of the kidney stained for macrophages by immunohistochemistry. Original magnification $\times 200$. (B) Analysis of macrophage infiltrate in WT (gray bars),

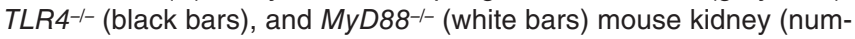
bers $/ 10$ HPFs). Data shown are mean \pm SD. $n=6-8$ per group. The comparison between $T L R 4^{-/-}$mice or MyD88 $8^{-/-}$mice and WT controls is indicated by asterisks. ${ }^{* *} P<0.01 ;{ }^{\#} P<0.001$.

We produced mice with TLR4 present on leukocytes but absent

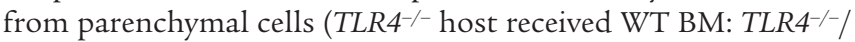
WTBM), and mice with TLR4 present on parenchymal cells but not

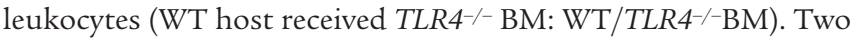
additional groups of mice were produced with TLR4 present on all cells (WT/WTBM) or with a complete absence of TLR4 (TLR4-1-/ $\left.T L R 4^{-/-} \mathrm{BM}\right)$. These sham chimeras were produced using methods identical to those used for the chimeric mice. Eight weeks after $\mathrm{BM}$ transplantation, chimeric mice were subjected to kidney ischemia. At that time, PCR analysis of genomic DNA extracted from leukocytes showed that in WT/TLR4-/-BM chimeras, 94\% $\pm 5.1 \%$ of genomic DNA was derived from the TLR4 $4^{--}$strain, and in TLR4-/-/WT chimeras, $91 \% \pm 6.8 \%$ of genomic DNA was derived from WT animals. WT/WTBM mice showed significant kidney dysfunction and injury at day 1 after ischemia/reperfusion, while TLR $4^{-/-} /$TLR $4^{-/-}$BM chimeric mice were protected from kidney IRI as measured by day 1 serum creatinine and tubular damage (Figure 13). Creatinine levels and tubular injury scores recapitulated those observed in WT and TLR4 ${ }^{-/}$mice (Figures 5 and 6), excluding an effect of the BM transplant procedure per se on the response to kidney ischemia. Moreover, TLR4-/-/WTBM chimeras were protected from kidney IRI to the same degree as TLR $4^{-/-} / T L R 4^{-/-} \mathrm{BM}$ mice, while WT/TLR4-/-BM mice enjoyed only partial protection as assessed by day 1 serum creatinine and tubular damage (Figure 13). These results suggest that functional TLR4 on kidney parenchymal cells makes the more significant contribution to kidney damage although leukocytes are clearly also important in IRI.

\section{Discussion}

Recent experimental data suggest that IRI rapidly activates innate immune responses. TLR4 has been shown to be upregulated in kidney IRI, particularly by TECs $(29,30)$. In this study, we found that expression of both TLR4 and a number of its endogenous ligands was increased following kidney ischemia and that the full development of kidney IRI was dependent upon signaling through the TLR4/MyD88 pathway. Mice genetically deficient in TLR4 or the adaptor molecule MyD88 were protected from kidney dysfunction and histological damage. Protection was associated with a reduction in proinflammatory cytokine and chemokine generation, a concomitant decrease in infiltration by macrophages and neutrophils, and a reduction in TEC apoptosis. We demonstrated that TLR4 signaling in kidney parenchymal cells made the more significant contribution to kidney damage although expression of TLR4 on leukocytes was clearly also important in IRI.

Within the kidney, both kidney parenchymal cells and BMderived cells express pattern recognition molecules, including members of the TLR system. Interstitial and glomerular macrophages express TLR1, $-2,-4$, and -6 , and DCs express TLR4, -7, -8, and -9, while TECs and mesangial cells express TLR1, $-2,-3,-4$, and -6 

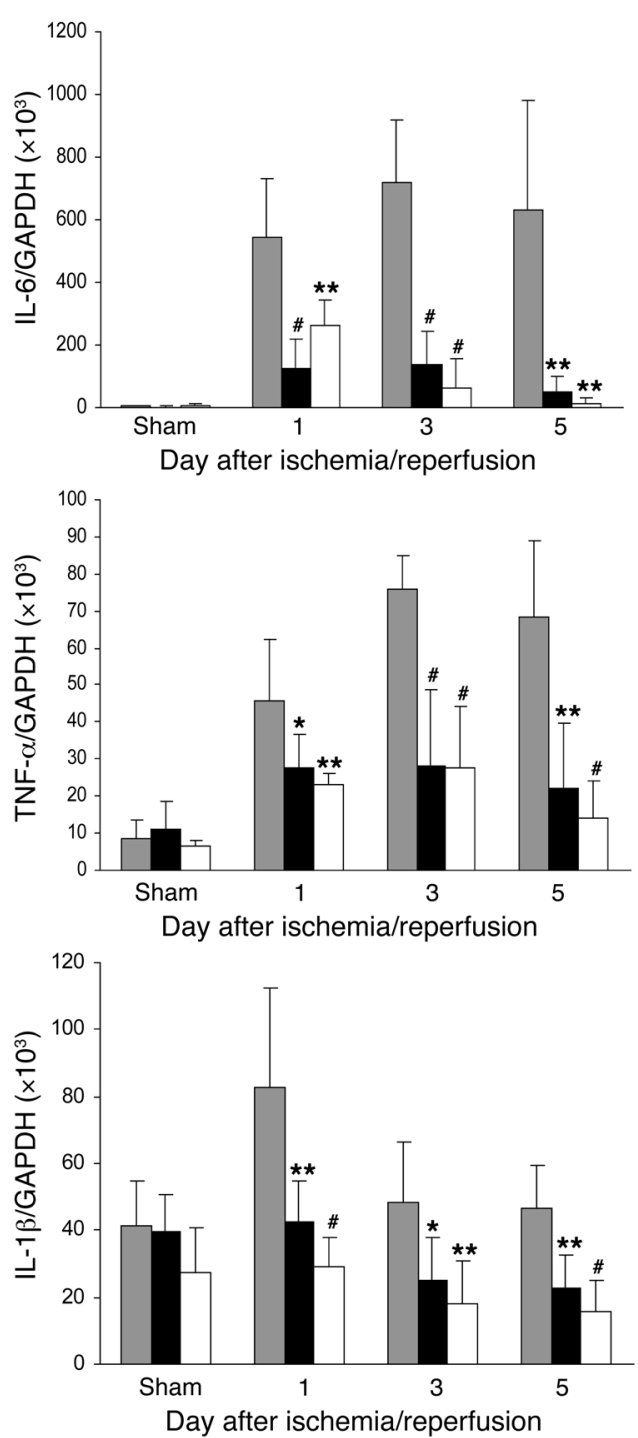

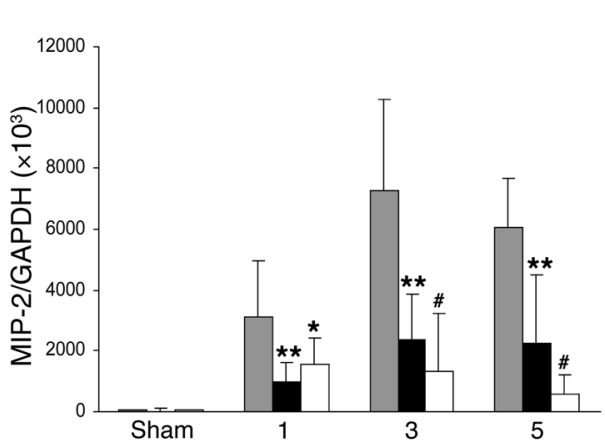

Day after ischemia/reperfusion

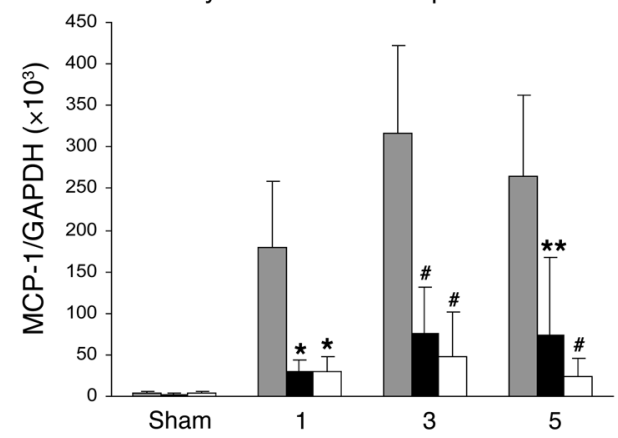

Day after ischemia/reperfusion

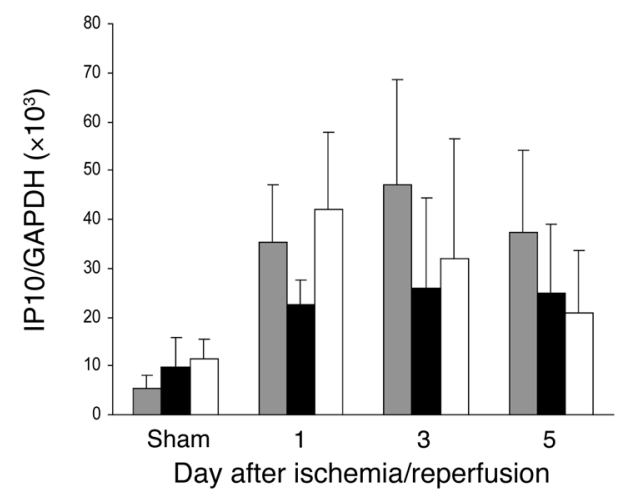

\section{Figure 9}

Proinflammatory cytokine and chemokine mRNA profile in the kidney measured by real-time PCR. mRNA expression of proinflammatory cytokines (IL-6, TNF- $\alpha$, and IL-1 $\beta$ ) and chemokines (MIP-2 and MCP-1) in the kidney was significantly reduced in TLR4 $4^{--}$(black bars) and $M y D 88^{-1-}$ (white bars) mice compared with WT controls (gray bars) from day 1 to day 5 after reperfusion. Results have been normalized by expressing the number of transcript copies as a ratio to GAPDH. Data shown are mean \pm SD. $n=6-8$ per group. The comparison between $\mathrm{TLR4}^{-/-}$mice or $\mathrm{MyD} 88^{-/-}$mice and WT controls is indicated by asterisks. ${ }^{*} P<0.05 ;{ }^{* *} P<0.01,{ }^{\#} P<0.001$.
$(15,32-34)$. Expression by other types of kidney parenchymal cells such as endothelial cells or fibroblasts is possible; however, this has not been reported. Kidney DCs have recently been shown to form an interdigitating network extending throughout the interstitium and also present within the glomerulus in mesangial areas $(35,36)$. Thus, kidneys are well equipped to respond to TLR ligands. The classical ligand for TLR4 is bacterial LPS, and TLR4, present on both immune cells and kidney parenchymal cells, is involved in pathological kidney responses in experimental models of kidney infection and systemic bacterial sepsis $(18,37)$.

Endogenous ligands have been identified for many TLRs (19, 20 ), and several of these ligands have been implicated in the pathogenesis of IRI. HMGB1, recently identified as an inflammatory cytokine $(38,39)$, can be released from necrotic or damaged cells as a signal to trigger inflammation (40). HMGB1 produced by ischemic hepatocytes binds to both TLR 2 and TLR4, and this interaction is critical for the development of lethal hepatic IRI. Hyaluronan fragments have been shown to signal through TLR4 and/or TLR2 on DCs and endothelial cells $(10,41)$ and can function as an endogenous adjuvant in alloresponses, inducing expression of costimulatory molecules and production of TNF- $\alpha$ by DCs, thus enhancing $\mathrm{T}$ cell priming (10). Chemokine and cytokine production by macrophages, kidney TECs, and lung epithelial cells mediated by the binding of hyaluronan to TLR4 has also been documented (42-44). Biglycan, an ECM component, can promote inflammation by signaling through TLR 2 and -4 on macrophages (24). In the current study, we demonstrated upregulation of several ligands, including HMGB1, hyaluronan (and its synthetic enzyme HAS), and biglycan in kidney during IRI, suggesting that any or all of these may act as endogenous ligands for TLR4 in our model. In contrast, expression of HSP70 was not increased at either the gene or the protein level. Our studies provide circumstantial evidence that endogenous ligands are the source of TLR4 activation during IRI. Further studies will be necessary to determine the time course of expression of the different ligands in response to ischemic insults of varying severity and to evaluate their relative contributions to the development of kidney IRI.

TLR4 engagement by its ligands triggers multiple downstream effects including the activation and expression of proinflammatory cytokines (TNF- $\alpha$, IL-1 $\beta$, and IL-6) and chemokines responsible for neutrophil (IL-8 and MIP-2) and macrophage (MCP-1) accumulation, all features of IRI. We were able to confirm the proposed 

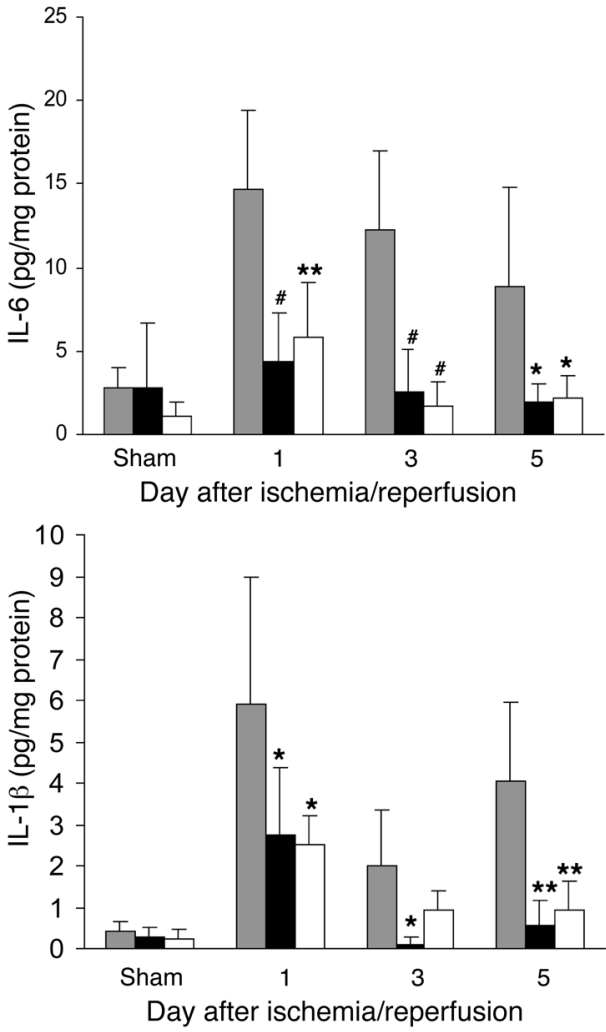
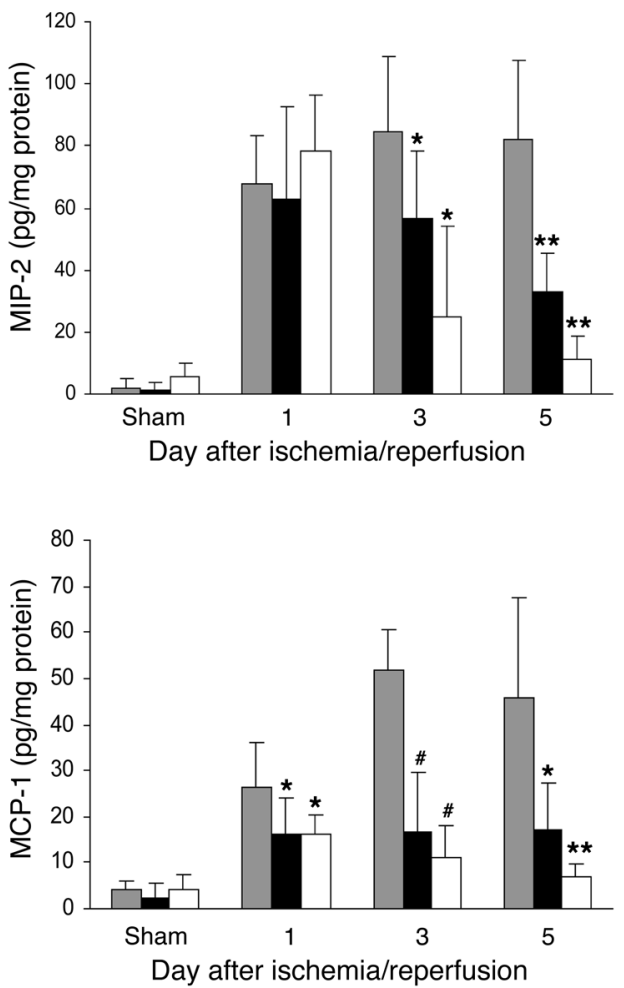

Figure 10

Cytokine and chemokine protein expression in the kidney measured by ELISA. Protein expression of cytokines (IL-6 and IL-1 $\beta$ ) and chemokines (MIP-2 and MCP-1) in the kidney was significantly reduced in $T L R 4^{-/-}$(black

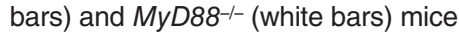
compared with WT controls (gray bars) from day 1 to day 5 after reperfusion. ${ }^{*} P<0.05,{ }^{* *} P<0.01,{ }^{*} P<0.001$. role of TLR4 in IRI by demonstrating that TLR4-deficient mice were protected against kidney IRI, with significantly lower serum creatinine, less tubular damage, and less interstitial neutrophil and macrophage accumulation versus WT controls. The major intracellular signaling pathway for TLR4 requires participation of the adaptor protein MyD88. Mice deficient in MyD88 were protected from kidney damage following IRI to an extent equal to TLR4deficient mice, suggesting the MyD88-dependent TLR4 pathway is responsible for TLR4-mediated kidney IRI. The impact of TLR4 deficiency on cytokine and chemokine expression and macrophage and neutrophil accumulation in IRI was also consistent with involvement of the MyD88 pathway. TLR4 signaling may also proceed via a MyD88-independent pathway involving the adaptor molecule TRIF, stimulating IFN-inducible genes such as IP10. In contrast to the expression pattern of MyD88-dependent cytokines and chemokines, we found no significant difference in IP10 mRNA expression in TLR4 $4^{--}, M y D 88^{-/-}$, and WT kidney at day 1 after IRI. While maintained expression of IP10 in the $M y D 88^{-/-}$ group is consistent with an intact TLR4/TRIF pathway in these mice, it is perhaps surprising that no reduction of IP10 expression was observed in the TLR4 $4^{--}$group. These results do not exclude a role for the TRIF pathway but do suggest that signaling via MyD88 is the dominant pathway leading to kidney injury. MyD88 is also required for signaling through TLR2, another receptor involved in the pathogenesis of kidney IRI (31). Genetic absence or knockdown of TLR2 resulted in reduced cytokine and chemokine production, reduced leukocyte infiltration, and protection from kidney dysfunction and tubular damage in the study of Leemans et al. (31). Given that both TLR2 and -4 have similar downstream effects following engagement $(10,23,42)$ and signal through a common adaptor molecule, MyD88, it is somewhat surprising that more redundancy between their effects was not observed and that both TLR2- and TLR4-deficient mice were significantly protected against kidney IRI. Moreover, in our study, MyD88-deficient mice, in which signaling through both TLR2 and -4 is affected, did not enjoy greater protection from IRI than TLR4 $4^{-/-}$mice. One possible explanation for these observations is that the influence of an individual TLR on the inflammatory response to ischemia may depend upon the profile of endogenous ligands produced by ischemia and that this profile may vary according to the duration and severity of the insult as well as the tissue involved. Although our study and that of Leemans et al. employed very similar models, the duration of kidney ischemia in the latter was considerably longer than that in our study (45 versus 22 minutes), and endogenous ligand expression, consequently, could differ as well.

Both BM-derived cells and parenchymal cells are known to contribute to inflammation and injury within the kidney in various conditions. To determine the relative contribution of TLR4 on kidney parenchymal cells and leukocytes to kidney damage in IRI, BM chimeric mice were generated. We demonstrated that during IRI, mice with TLR4-deficient leukocytes but TLR4-competent parenchymal cells developed significantly more kidney dysfunction and damage compared with chimeras with TLR4-deficient parenchymal cells but competent leukocytes, suggesting functional TLR4 on kidney parenchymal cells makes the more significant contribution to IRI. Consistent with this, our in vitro results confirmed that kidney tubular cells required TLR4 to significantly upregulate proinflammatory cytokines and chemokines following ischemia and that in the absence of TLR4 or MyD88, they were protected against undergoing apoptosis. Similar consequences of TLR2 signaling in ischemic kidney tubular cells were also reported by Leemans et al. (31). In addition to tubular cells, other TLR4-expressing intrin- 
A

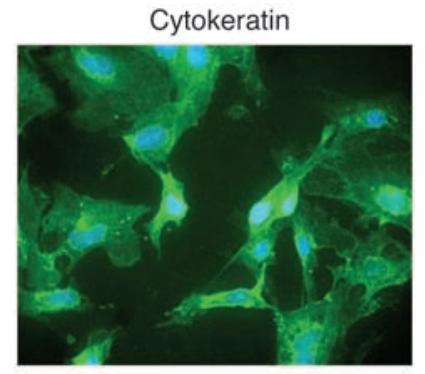

B
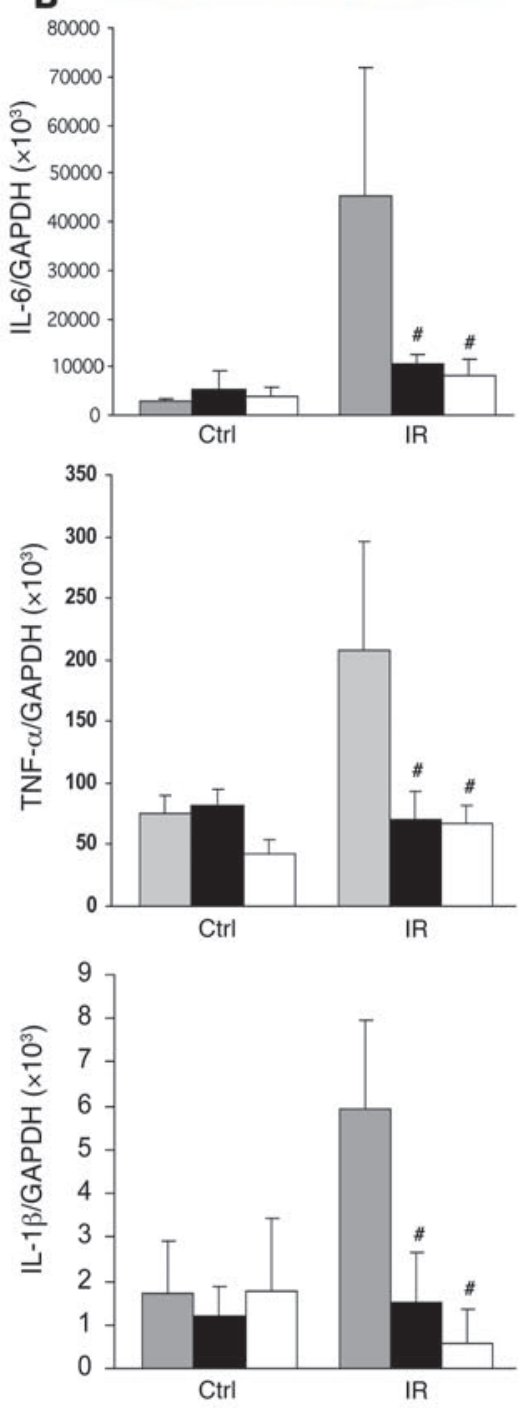
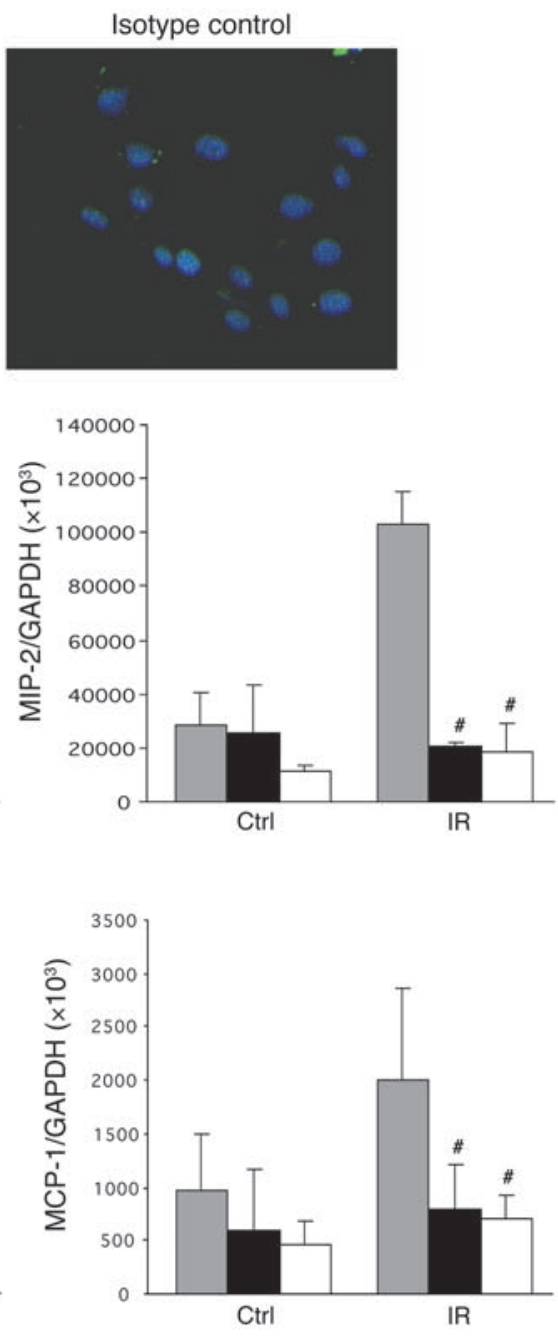

\section{Figure 11}

Proinflammatory cytokine and chemokine gene expression in primary cultured TECs submitted to 1 hour ischemia in vitro. (A) Immunofluorescence staining of primary cultured TECs from C57BL/6 mice with mAbs against cytokeratin (green) and nuclear staining with DAPI in blue. Original magnification, $\times 400$. (B) mRNA expression of proinflammatory cytokines (IL- 6, IL-1 $\beta$, and TNF- $\alpha$ ) and chemokines (MIP-2 and MCP-1) in TLR4-/- and $M y D 88^{-/-}$primary cultured TECs submitted to 1 hour ischemia in vitro was significantly reduced at 1 hour after medium replacement as compared with WT controls. The results have been normalized by expressing the number of transcript copies as a ratio to GAPDH. These data are representative of 3 experiments. The comparison between $\mathrm{TLR}^{-{ }^{--}}$or $M y D 88^{-1-}$ TECs and WT controls is indicated by asterisks. ${ }^{P}<0.001$.

WT controls. This was not seen in TLR2 ${ }^{-/-}$mice in IRI (31), whereas TLR2 ligation on both BMderived and parenchymal kidney cells plays a role in amplifying the inflammatory effects in immune-mediated glomerulonephritis $(45,46)$, another form of sterile tissue injury. In contrast, nonparenchymal cells are the critical cell type in the pathogenesis of TLR-mediated liver IRI (26).

In summary, our results document the important role of TLR4- and MyD88-mediated signaling in the pathogenesis of kidney IRI and suggest that this pathway is central in the innate immune response that leads to kidney injury.

\section{Methods}

Animals. Male WT (C57BL/6) mice (10-12 weeks old) were obtained from the Animal Resource Center (Perth, Western Australia, Australia). TLR $4^{-/-}$and $M y D 88^{-/-}$ mice were kindly provided by D. Hume (University of Queensland, Brisbane, Queensland, Australia) and W. Health (The Walter and Eliza Hall Institute of Medical Research, Melbourne, Victoria, Australia) with permission from S. Akira (Osaka University, Osaka, Japan) and were backcrossed more than 9 times onto a C57BL/6 background. The mice were housed in individual microisolator cages with free access to sterile acidified water and irradiated food in a specific pathogen-free facility at the University of Sydney. Male mice weighing 25-30 g were used in all experiments. Experiments were conducted by following established guidelines

sic kidney cell types, such as endothelial cells, may be contributing to IRI. While we have not specifically addressed the role of endothelial cells, our in vitro data do suggest that TLR4 on kidney tubular cells plays a significant role in ischemia-mediated injury. The kidney is richly supplied with a network of resident DCs and macrophages $(35,36)$, and these, as well as infiltrating leukocytes, could promote IRI through the elaboration of soluble mediators and through cell-cell contact. Consistent with this, TLR4 expression by leukocytes also appears important in IRI, as chimeric mice lacking TLR4 on BM-derived cells alone were partially protected from kidney dysfunction and tubular injury as compared with for animal care and were approved by the animal ethics committee of the University of Sydney.

Induction of kidney IRI. Mice were anesthetized with inhalational isoflurane (Abbott Australasia Pty.). Using a midline abdominal incision, both renal pedicles were clamped for 22 minutes with microaneurysm clamps. During the period of ischemia, body temperature was maintained by placing the mice on a $37^{\circ} \mathrm{C}$ heating pad. After removal of the clamps, the kidneys were inspected for 1 minute for restoration of blood flow, returning to their original color. The abdomen was closed. Sham-operated mice $(n=5$ per group) received identical surgical procedures except that microaneurysm clamps were not applied; these were sacrificed 1 day after operation. 

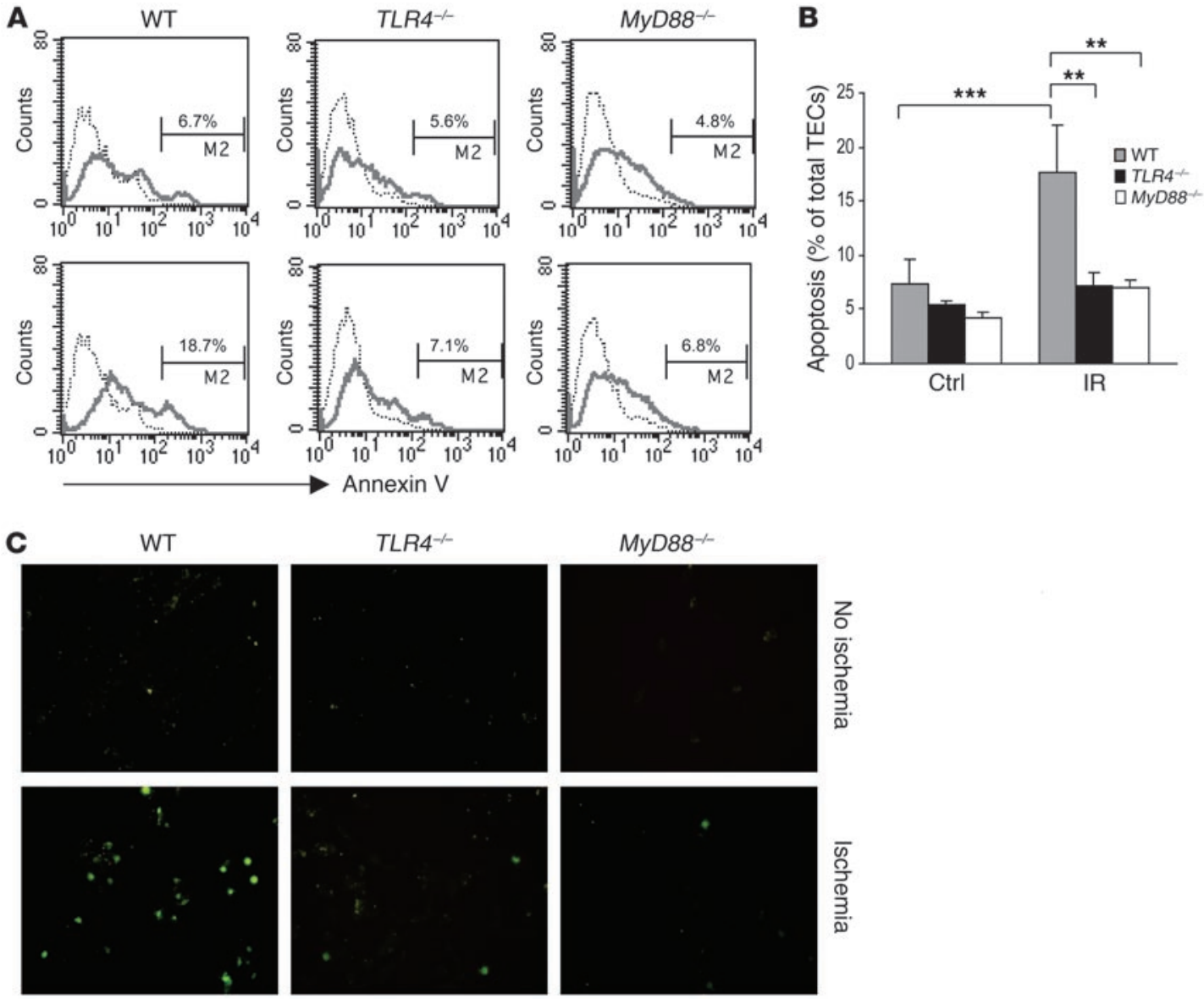

Figure 12

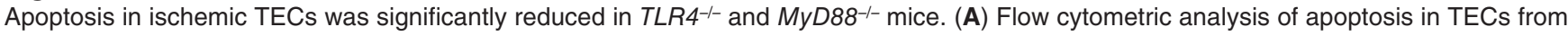

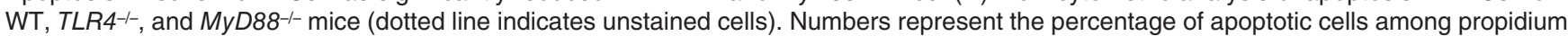
iodide-negative, viable cells. TECs subjected to ischemia are represented in the lower panels compared with nonischemic controls in the upper panels. (B) In WT mice, the proportion of apoptotic cells increased from $6.7 \%$ to $18.7 \%$ after ischemia. In contrast, the proportion of apoptotic

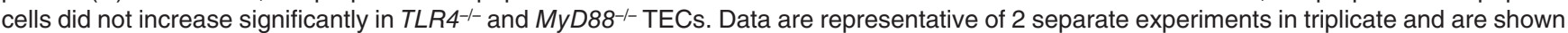
as mean \pm SD. ${ }^{* *} P<0.01$; ${ }^{* *} P<0.001$. (C) Apoptosis was further confirmed using a TUNEL assay. TECs subjected to ischemia are shown in the lower panels, with nonischemic controls in the upper panels. Original magnification, $\times 200$.

To maintain fluid balance, all mice were supplemented with $1 \mathrm{ml}$ of saline administered subcutaneously. Mice were sacrificed 1, 3, 5, and 9 days after reperfusion ( $n=6-8$ per group). Blood was collected and kidney tissues were divided up to be either snap frozen for subsequent mRNA extraction, embedded and frozen in OCT compound (Sakura), or fixed in 10\% neutralbuffered formalin for paraffin embedding.

Assessment of kidney function. Serum creatinine level was measured using the modified Jaffe rate reaction by the Department of Biochemistry, Royal Prince Alfred Hospital.

Primary culture of mouse renal TECs. Primary mouse renal TECs were generated following the method described by Wuthrich et al. (47). In brief, kidneys were flushed with saline in vivo to remove blood cells, then removed. The kidney cortices from WT, TLR4 $4^{-/}$, and $M y D 88^{-/-}$mice were cut into pieces of approximately $1 \mathrm{~mm}^{3}$ and then digested in HBSS containing 3 $\mathrm{mg} / \mathrm{ml}$ of collagenase at $37^{\circ} \mathrm{C}$ for 25 minutes and washed in DMEM/F12 medium (Invitrogen). The kidney digest was washed through a series of sieves (mesh diameters of $250,150,75$, and $40 \mu \mathrm{m}$ ). The cortical tubular cells were spun down at $300 \mathrm{~g}$ for 5 minutes and further washed. The cell pellet was resuspended in defined K1 medium (47): DMEM/F12 medium supplemented with $25 \mathrm{ng} / \mathrm{ml}$ epidermal growth factor, $1 \mathrm{ng} / \mathrm{ml} \mathrm{PGE}_{1}$, $5 \times 10^{-11} \mathrm{M}$ triiodothyronine, $5 \times 10^{-8} \mathrm{M}$ hydrocortisone (Sigma-Aldrich), insulin-transferrin-sodium selenite media supplement, $1 \%$ penicillin/ streptomycin, $25 \mathrm{mM}$ HEPES, and 5\% FCS (Invitrogen). The cell suspension was then placed on cell culture Petri dishes and incubated at $37^{\circ} \mathrm{C}$ for 2-3 hours to facilitate adherence of contaminating glomeruli. The nonadherent tubules were then collected and cultured on collagen-coated Petri dishes (BD Biosciences) in K1 medium until epithelial colonies were established. Expression of the epithelial cell marker cytokeratin was verified by immunofluorescent staining with an anti-cytokeratin antibody (SigmaAldrich). Cells were $96 \%$ to $100 \%$ cytokeratin positive. Experiments were commenced after the cells had reached $90 \%$ confluence, which was usually between 5-7 days after the isolation procedure.

Induction of renal TEC ischemia in vitro. Renal TECs were rendered transiently ischemic by immersing the cellular monolayer in mineral oil according to the protocol of Meldrum et al. (48). This immersion induced simulated ischemia by restricting cellular exposure to oxygen and nutrients as well as by limiting metabolite washout. Renal TECs were placed in serum-free K1 medium for 24 hours, washed twice with PBS, and immersed in mineral 
A

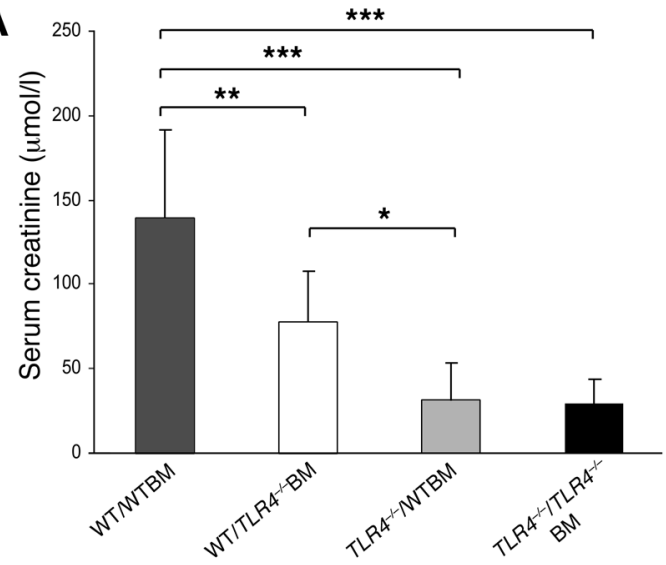

B

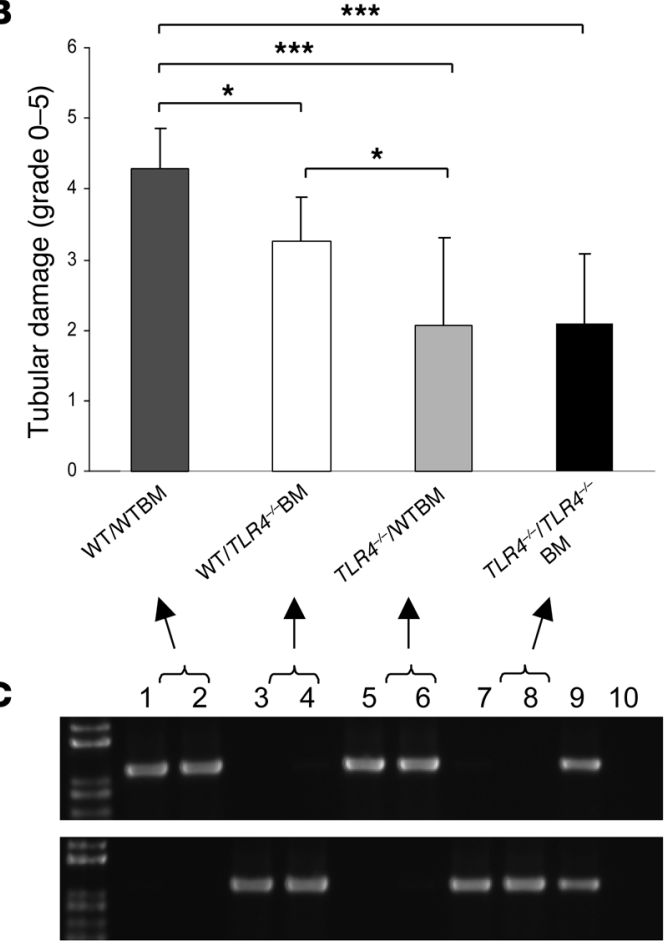

oil (Sigma-Aldrich) for 60 minutes at $37^{\circ} \mathrm{C}$. After extensive washing with PBS, cells were incubated in $\mathrm{K} 1$ medium and then collected at 1 hour after medium replacement for cytokine and chemokine mRNA expression and at 16 hours after medium replacement for detection of apoptosis. TECs were exposed to serum-free K1 medium alone as the nonischemic control.

Analysis of apoptosis. Apoptosis of TECs in culture was measured using FACS with annexin V-FITC staining following the manufacturer's protocol (BD Biosciences). In brief, monolayers of TEC were released by a brief incubation with trypsin-EDTA solution (Invitrogen) and then incubated with annexin V-FITC and propidium iodide in $1 \times$ binding buffer for 15 minutes. All samples were analyzed on a FACSCalibur flow cytometer using CellQuest software (BD). The apoptosis experiments were performed in triplicate on 2 separate occasions.

The proportion of apoptotic cells in TECs culture was also confirmed by terminal transferase-mediated dUTP nick-end labeling (TUNEL) assay following the manufacturer's protocol. In brief, cells were grown to confluence in 4-well chamber slides, fixed in 4\% paraformaldehyde in PBS,

\section{Figure 13}

Functional TLR4 on intrinsic kidney cells makes the more significant contribution to kidney damage. WT/WTBM mice showed significant kidney dysfunction and injury at day 1 after ischemia/reperfusion, while TLR4 $4^{-/} / T L R 4^{-/-}$BM chimeric mice were protected from kidney IRI as measured by day 1 serum creatinine (A) and tubular damage (B). Creatinine levels and tubular injury scores replicated those observed in WT and TLR4 $4^{--}$mice (Figures 5 and 6), excluding an effect of the $\mathrm{BM}$ transplant procedure per se on the response to renal ischemia. Moreover, TLR4--/WTBM chimeras were protected from kidney IRI

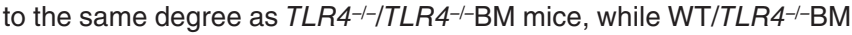
mice enjoyed only partial protection as assessed by day 1 serum creatinine and tubular damage (A and $\mathbf{B})$. Data shown are mean \pm SD. $n=7-10$ per group. ${ }^{\star} P<0.05,{ }^{\star \star} P<0.01,{ }^{\star \star \star} P<0.001$. Full replacement of hematopoietic cells in the chimeric mice was confirmed by genotyping of genomic DNA from whole blood using PCR. PCR products shown on representative gels $(\mathbf{C})$. The top panel represents PCR products for the WT allele DNA, and the bottom panel represents PCR products for the mutated allele DNA (lanes 1-2: WT/WTBM; lanes 3-4: WT/TLR4---BM; lanes 5-6: TLR4--/WTBM; lanes 7-8: TLR4--/ TLR4-/-BM; lane 9: TLR4 heterozygous blood as positive controls; and lane 10: negative controls).

and then incubated with $0.1 \%$ Triton X-100 in $0.1 \%$ sodium citrate. DNA fragmentation in apoptotic cells was labeled and identified by terminal transferase dUTP conjugated with fluorescein (Roche Diagnostics). The samples were viewed under a fluorescence microscope and photographed.

Histological examination. Kidneys embedded in paraffin were sectioned at $3 \mu \mathrm{m}$ and stained with H\&E by standard methods. Markers of tubular damage were scored by calculation of the percentage of tubules in the corticomedullary junction that displayed cell necrosis, loss of the brush border, cast formation, and tubular dilatation as follows (49): 0 , none; $1, \leq 10 \% ; 2$, $11-25 \%$; 3, 26-45\%; 4, 46-75\%; and 5, >76\%. Histological examination was performed by 2 blinded observers. At least 10 high-power fields (HPFs) (magnification, $\times 200$ ) per section for each sample were examined.

Immunostaining. Formalin-fixed paraffin-embedded sections of $5-\mu \mathrm{m}$ thickness were deparaffinized and boiled for 10 minutes in $10 \mathrm{mM}$ sodium citrate buffer ( $\mathrm{pH}$ 6.0) for neutrophil detection or digested with $0.5 \mathrm{mg} /$ $\mathrm{ml}$ of protease (50) (Sigma-Aldrich) for TLR4 and hyaluronan detection. Frozen sections were cut to a thickness of $7 \mu \mathrm{m}$ and fixed in acetone for macrophage detection. Immunohistochemistry was performed using the following primary antibodies: rat anti-mouse neutrophil (clone 4/7), macrophage F4/80 (AbD Serotec), and goat anti-mouse TLR4 (SC-12511; Santa Cruz Biotechnology Inc.). Concentration-matched rat IgG or goat IgG was used as an isotype-negative control. Preabsorption of goat anti-mouse TLR4 polyclonal antibody with a specific blocking peptide (SC-12511P; Santa Cruz Biotechnology) was also used as a negative control for TLR4 staining. The sections were blocked with $10 \%$ normal horse serum for 20 minutes and incubated with primary antibodies for 60 minutes. Sections were exposed to $3 \% \mathrm{H}_{2} \mathrm{O}_{2}$ in methanol for 5 minutes to quench endogenous peroxidases, then incubated with the biotinylated secondary antibody anti-rat IgG (BD Biosciences - Pharmingen) or biotinylated anti-goat IgG (Vector Laboratories). Instead of a primary antibody, a b-HABP (Seikagaku Corp.) at $5 \mu \mathrm{g} / \mathrm{ml}$ was used for hyaluronan staining. VECTASTAIN ABC kit (Vector Laboratories) was then applied according to the manufacturer's instructions, followed by 3,3'-diaminobenzidine (DAB) substrate-chromogen solution (Dako). The slides were counterstained with Harris hematoxylin.

For HMGB1 immunofluorescent staining, frozen sections were blocked with $1 \%$ BSA in PBS for 20 minutes and incubated with rabbit polyclonal antibodies to HMGB1 (Abcam) for 60 minutes followed by anti-rabbit IgG conjugated with Alexa Fluor 488 (Invitrogen). 
Quantification of immunohistochemistry. Analysis of the cellular infiltrate was performed in a blinded manner by assessing 20 consecutive HPFs (magnification, $\times 400$ ) of the outer medulla and corticomedullary junction on each section. Using an ocular grid, the number of cells staining positively for each antibody were counted and expressed as cells per $10 \mathrm{HPFs}$. Tubular TLR4 expression was scored by calculation of the percentage of positive-staining tubules in the corticomedullary junction as follows: 0 , none; $1, \leq 10 \% ; 2,11 \%-25 \% ; 3,26 \%-45 \% ; 4,46 \%-75 \%$; and $5,>76 \%$. At least 10 consecutive HPFs (magnification, $\times 200$ ) per section were scored by 2 blinded operators. An area of $0.45 \mathrm{~mm}^{2}$ in the corticomedullary junction was analyzed for interstitial hyaluronan positive staining using a digital image analysis program (ACIS III Automated Cellular Imaging System; Dako). Ten areas in each section were analyzed for both area and intensity of staining. The results were expressed as a percentage of the analyzed area and an intensity score of positive staining.

Extraction of RNA and cDNA synthesis. Total RNA was extracted from renal tissue using TRIzOL (Invitrogen) according to the manufacturer's instructions. cDNA was synthesized using oligo d(T) ${ }_{16}$ (Applied Biosystems) and the SuperScript III Reverse Transcriptase Kit (Invitrogen) following a standard protocol.

Real-time PCR. Specific TaqMan primers and probes for TLR4 (Mm00445274_ml), IL-6 (Mm00446190_m1), TNF- $\alpha$ (Mm00443258_m1), IP10(Mm00445235_ml), Biglycan (Mm00455918_m1), HAS1 (Mm00468496_ml), and HSP70 (Mm0043069_sl) were obtained from Applied Biosystems. Specific Taqman primers and probes for MCP-1 (forward, 5'-GAGCATCCACGTGTTGGCT-3'; reverse, 5'-TGGTGAATGAGTAGCAGCAGGT-3'; probe, 6FAM-AGCCAGATGCAGTTAACGCCCCACTTAMRA); MIP-2 (forward, 5'-GCCCCCAGGACCCCA-3'; reverse, 5'-CTTTTTGACCGCCCTTGAGA-3'; probe, 6FAM-TGCGCCCAGACAGAAGTCATAGCCA-TAMRA); IL-1 $\beta$ (forward, $5^{\prime}$-GCACACCCACCCTGCAG-3'; reverse, 5'-AACCGCTTTTCCATCTTCTTCTT-3'; probe, 6FAMTGGAGAGTGTGGATCCCAAGCAATACCC-TAMRA); HMGB1 (forward, 5'-TGGGCGACTCTGTGCCTC-3'; reverse, 5'-GCCTCTCGGCTTTTTAGGATC-3'; probe, 6FAM-CGGAGGAAAATCAACTAAACATGGGCAAATAMRA); HAS2 (forward, 5'-CAGCCTTCAGAGCACTGGG-3'; reverse, 5'-TGAGGCAGGGTCAAGCATAGT-3'; probe, 6FAM-CGAAGCGTGGATTATGTACAGGTGTGTGACTC-TAMRA); and HAS3 (forward, 5'-ACTGCCTTCAAGGCCCTTG-3'; reverse, 5'-TGGGTCCAGCACAGTGTCAG-3'; probe, 6FAM-CAACTCAGTGGACTACATCCAGGTGTGTGAC-3') were designed using Primer Express Software (Applied Biosystems) and obtained from Sigma-Aldrich. Primers and a probe for GAPDH were used as previously described (51). cDNA was amplified in $1 \times$ Universal Master Mix (Applied Biosystems) with gene-specific primers and probe on the ABI Prism 7700 Sequence Detection System (Applied Biosystems), according to the manufacturer's instructions. Thermal cycling conditions were $50^{\circ} \mathrm{C}$ for 2 minutes and $95^{\circ} \mathrm{C}$ for 10 minutes, followed by 45 cycles of $95^{\circ} \mathrm{C}$ for 15 seconds and $60^{\circ} \mathrm{C}$ for 1 minute. Data were analyzed using the Sequence Detector V1.9 Analysis Software (Applied Biosystems). Expression of each gene was normalized against mRNA expression of the housekeeping gene GAPDH, as has previously been described $(51,52)$. Real-time PCR experiments for each gene were performed on 3 separate occasions.

Kidney tissue protein extraction for cytokine and chemokine measurements. Snap-frozen kidney tissue was added into prechilled CelLytic MT reagent (Sigma-Aldrich) with a $1 \%$ protease inhibitor cocktail (Sigma-Aldrich) for use with mammalian tissue extracts and then homogenized. The samples were incubated for 30 minutes at $4{ }^{\circ} \mathrm{C}$ and centrifuged at $16,000 \mathrm{~g}$ at $4^{\circ} \mathrm{C}$ for 15 minutes to pellet the tissue debris. The supernatant was stored at $-70^{\circ} \mathrm{C}$. Protein concentrations were determined by a colorimetric protein assay (Bio-Rad) using protein standards from Sigma-Aldrich.

ELISA. Cytokines and chemokines were measured in kidney homogenates using ELISA kits according to the manufacturers' instructions. Kits for IL-6, IL- $1 \beta$, TNF- $\alpha$, and MIP- 2 were obtained from R\&D Systems, and the MCP- 1 ELISA kit was obtained from BD Biosciences. Protein levels of cytokines and chemokines were corrected for the total amounts of protein, and the results were expressed as $\mathrm{pg} / \mathrm{ml}$. The detection limits were $7.8 \mathrm{pg} / \mathrm{ml}$ for IL-6, IL- $1 \beta$, and MIP-2; $46.9 \mathrm{pg} / \mathrm{ml}$ for TNF- $\alpha$; and $15.6 \mathrm{pg} / \mathrm{ml}$ for MCP-1.

Western blot. HSP70 protein expression in kidney tissue was confirmed by Western blot analysis. Aliquots $(50 \mu \mathrm{g})$ of kidney homogenates were separated on $10 \%$ polyacrylamide gel (Sigma-Aldrich) and transferred to a PVDF membrane (PerkinElmer). The membrane was blocked overnight in Western blocker solution (Sigma-Aldrich), incubated with anti-HSP70 antibody (Nventa) in Western Blocker Solution (Sigma-Aldrich) for 1 hour, washed, incubated with anti-mouse IgG conjugated with HRP (SigmaAldrich), then washed. Positive bands were detected by chemiluminescence technology (Sigma-Aldrich) using the G:Box gel documentation and analysis system (Syngene). The membrane was also probed with anti- $\beta$-actin antibody (Sigma-Aldrich) for $\beta$-actin expression.

Generation of BM chimeric mice. BM cells were collected from WT or TLR4-/ mice by flushing femurs and tibiae with RPMI 1640 containing 5\% FCS. Chimeric mice were produced by transfer of donor BM cells into irradiated recipient animals using the following recipient/donor combinations of WT and TLR4 $4^{--}$mice $\left(n=8-10\right.$ per group): WT/WTBM, WT/TLR4 ${ }^{-/-B M}$,

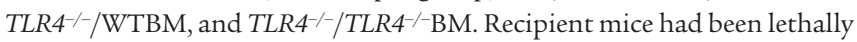
irradiated with a signal dose of $9.5 \mathrm{~Gy}$ from a cesium source using a $137 \mathrm{Cs}$ irradiator (Gammacell 40 Exactor; Nordion International Inc.). Six hours after irradiation, recipient irradiated mice received $8 \times 10^{6} \mathrm{BM}$ cells via the tail vein. The animals were allowed to recover for $8-10$ weeks to ensure stable engraftment before being subjected to 22 minutes of kidney ischemia and 24-hour reperfusion. Full chimerism of each mouse was confirmed by genotyping of genomic DNA from peripheral blood using REDExtract-NAmp Blood PCR kits (Sigma-Aldrich) following a standard protocol. The PCR products were run on $1 \%$ agarose gel, and band density was quantified by gel documentation and analysis system (Syngene).

Statistics. Results are expressed as mean \pm SD. Multiple groups were compared using 1-way ANOVA with post-hoc Bonferroni's correction (GraphPad Prism 4.0; GraphPad Software). Two groups (Figure 1B) were compared using an unpaired Student's $t$ test (2-tailed). A value of $P<0.05$ was considered significant.

\section{Acknowledgments}

This work was supported by the National Health and Medical Research Council (NHMRC) of Australia (project grant 402539).

Received for publication November 20, 2006, and accepted in revised form June 28, 2007.

Address correspondence to: Huiling Wu, Collaborative Transplant Research Group, Room W607, Blackburn Building D06, University of Sydney, Sydney, New South Wales 2006, Australia. Phone: 612-93512898; Fax: 612-9351-8771; E-mail: huilingw@med.usyd.edu.au.
1. Herrero-Fresneda, I., et al. 2003. Do alloreactivity and prolonged cold ischemia cause different elementary lesions in chronic allograft nephropathy? Am. J. Pathol. 162:127-137.

2. Tilney, N.L., and Guttmann, R.D. 1997. Effects of initial ischemia/reperfusion injury on the transplanted kidney. Transplantation. 64:945-947.

3. Chapman, J.R., O'Connell, P.J., and Nankivell, B.J. 2005. Chronic renal allograft dysfunction. J. Am. Soc. Nephrol. 16:3015-3026.
4. Bonventre, J.V., and Zuk, A. 2004. Ischemic acute renal failure: an inflammatory disease? Kidney Int. 66:480-485.

5. Jo, S.K., Sung, S.A., Cho, W.Y., Go, K.J., and Kim, H.K. 2006. Macrophages contribute to the initia- 
tion of ischaemic acute renal failure in rats. Nephrol. Dial. Transplant. 21:1231-1239.

6. Zhou, W., et al. 2000. Predominant role for C5b-9 in renal ischemia/reperfusion injury. J. Clin. Invest. 105:1363-1371.

7. de Vries, B., et al. 2003. Complement factor C5a mediates renal ischemia-reperfusion injury independent from neutrophils. J. Immunol. 170:3883-3889.

8. Boros, P., and Bromberg, J.S. 2006. New cellular and molecular immune pathways in ischemia/reperfusion injury. Am. J. Transplant. 6:652-658.

9. Chalasani, G., et al. 2004. The allograft defines the type of rejection (acute versus chronic) in the face of an established effector immune response. J. Immunol. 172:7813-7820.

10. Tesar, B.M., et al. 2006. The role of hyaluronan degradation products as innate alloimmune agonists. Am. J. Transplant. 6:2622-2635.

11. Medzhitov, R., Preston-Hurlburt, P., and Janeway, C.A., Jr. 1997. A human homologue of the Drosophila Toll protein signals activation of adaptive immunity. Nature. 388:394-397.

12. Matzinger, P. 2002. The danger model: a renewed sense of self. Science. 296:301-305.

13. Gordon, S. 2002. Pattern recognition receptors: doubling up for the innate immune response. Cell. 111:927-930.

14. Medzhitov, R., and Janeway, C.A., Jr. 2002. Decoding the patterns of self and nonself by the innate immune system. Science. 296:298-300.

15. Anders, H.J., Banas, B., and Schlondorff, D. 2004. Signaling danger: toll-like receptors and their potential roles in kidney disease. J. Am. Soc. Nephrol. 15:854-867.

16. Yamamoto, M., Takeda, K., and Akira, S. 2004. TIR domain-containing adaptors define the specificity of TLR signaling. Mol. Immunol. 40:861-868.

17. Jones, B.W., Heldwein, K.A., Means, T.K., Saukkonen, J.J., and Fenton, M.J. 2001. Differential roles of Toll-like receptors in the elicitation of proinflammatory responses by macrophages. Ann. Rheum. Dis. 60(Suppl. 3):iii6-iii12.

18. Cunningham, P.N., Wang, Y., Guo, R., He, G., and Quigg, R.J. 2004. Role of Toll-like receptor 4 in endotoxin-induced acute renal failure. J. Immunol. 172:2629-2635.

19. Marshak-Rothstein, A. 2006. Toll-like receptors in systemic autoimmune disease. Nat. Rev. Immunol. 6:823-835.

20. Beg, A.A. 2002. Endogenous ligands of Toll-like receptors: implications for regulating inflammatory and immune responses. Trends Immunol. 23:509-512.

21. Vabulas, R.M., et al. 2002. HSP70 as endogenous stimulus of the Toll/interleukin-1 receptor signal pathway. J. Biol. Chem. 277:15107-15112.

22. Vabulas, R.M., et al. 2001. Endocytosed HSP60s use toll-like receptor 2 (TLR2) and TLR4 to activate the toll/interleukin-1 receptor signaling pathway in innate immune cells. J. Biol. Chem. 276:31332-31339.

23. Yu, M., et al. 2006. HMGB1 signals through toll-like receptor (TLR) 4 and TLR2. Shock. 26:174-179.

24. Schaefer, L., et al. 2005. The matrix component biglycan is proinflammatory and signals through Toll-like receptors 4 and 2 in macrophages. J. Clin. Invest. 115:2223-2233. doi:10.1172/JCI23755.

25. Tsung, A., et al. 2005. The nuclear factor HMGB1 mediates hepatic injury after murine liver ischemiareperfusion. J. Exp. Med. 201:1135-1143.

26. Zhai, Y., et al. 2004. Cutting edge: TLR4 activation mediates liver ischemia/reperfusion inflammatory response via IFN regulatory factor 3-dependent MyD88-independent pathway. J. Immunol. 173:7115-7119.

27. Wu, H.S., et al. 2004. Toll-like receptor 4 involvement in hepatic ischemia/reperfusion injury in mice. HBPD INT. 3:250-253.

28. Oyama, J., et al. 2004. Reduced myocardial ischemia-reperfusion injury in toll-like receptor 4-deficient mice. Circulation. 109:784-789.

29. Wolfs, T.G., et al. 2002. In vivo expression of Tolllike receptor 2 and 4 by renal epithelial cells: IFNgamma and TNF-alpha mediated up-regulation during inflammation. J. Immunol. 168:1286-1293.

30. Kim, B.S., et al. 2005. Ischemia-reperfusion injury activates innate immunity in rat kidneys. Transplantation. 79:1370-1377.

31. Leemans, J.C., et al. 2005. Renal-associated TLR2 mediates ischemia/reperfusion injury in the kidney. J. Clin. Invest. 115:2894-2903. doi:10.1172/ JCI22832.

32. Tsuboi, N., et al. 2002. Roles of toll-like receptors in C-C chemokine production by renal tubular epithelial cells. J. Immunol. 169:2026-2033.

33. Patole, P.S., et al. 2006. Expression and regulation of Toll-like receptors in lupus-like immune complex glomerulonephritis of MRL-Fas(lpr) mice. Nephrol. Dial. Transplant. 21:3062-3073.

34. Wornle, M., et al. 2006. Novel role of toll-like receptor 3 in hepatitis $\mathrm{C}$-associated glomerulonephritis. Am. J. Pathol. 168:370-385.

35. Soos, T.J., et al. 2006. CX3CR1+ interstitial dendritic cells form a contiguous network throughout the entire kidney. Kidney Int. 70:591-596.

36. Kruger, T., et al. 2004. Identification and functional characterization of dendritic cells in the healthy murine kidney and in experimental glomerulonephritis. J. Am. Soc. Nephrol. 15:613-621.

37. Patole, P.S., et al. 2005. Toll-like receptor-4: renal cells and bone marrow cells signal for neutrophil recruitment during pyelonephritis. Kidney Int. 68:2582-2587.

38. Wang, H., et al. 1999. HMG-1 as a late mediator of endotoxin lethality in mice. Science. 285:248-251.

39. Yang, H., Wang, H., Czura, C.J., and Tracey, K.J. 2005. The cytokine activity of HMGB1. J. Leukoc. Biol. 78:1-8.

40. Scaffidi, P., Misteli, T., and Bianchi, M.E. 2002. Release of chromatin protein HMGB1 by necrotic cells triggers inflammation. Nature. 418:191-195.

41. Termeer, C., et al. 2002. Oligosaccharides of hyaluronan activate dendritic cells via toll-like receptor 4 . J. Exp. Med. 195:99-111.

42. Jiang, D., et al. 2005. Regulation of lung injury and repair by Toll-like receptors and hyaluronan. Nat. Med. 11:1173-1179.

43. Beck-Schimmer, B., Oertli, B., Pasch, T., and Wuthrich, R.P. 1998. Hyaluronan induces monocyte chemoattractant protein-1 expression in renal tubular epithelial cells. J. Am. Soc. Nephrol. 9:2283-2290.

44. Wuthrich, R.P. 1999. The proinflammatory role of hyaluronan-CD44 interactions in renal injury. Nephrol. Dial. Transplant. 14:2554-2556.

45. Brown, H.J., Lock, H.R., Sacks, S.H., and Robson, M.G. 2006. TLR2 stimulation of intrinsic renal cells in the induction of immune-mediated glomerulonephritis. J. Immunol. 177:1925-1931.

46. Brown, H.J., Sacks, S.H., and Robson, M.G. 2006. Toll-like receptor 2 agonists exacerbate accelerated nephrotoxic nephritis. J. Am. Soc. Nephrol. 17:1931-1939.

47. Wuthrich, R.P., et al. 1990. MHC class II, antigen presentation and tumor necrosis factor in renal tubular epithelial cells. Kidney Int. 37:783-792.

48. Meldrum, K.K., Meldrum, D.R., Hile, K.L., Burnett, A.L., and Harken, A.H. 2001. A novel model of ischemia in renal tubular cells which closely parallels in vivo injury. J. Surg. Res. 99:288-293.

49. Yamada, K., Miwa, T., Liu, J., Nangaku, M., and Song, W.C. 2004. Critical protection from renal ischemia reperfusion injury by CD55 and CD59. J. Immunol. 172:3869-3875.

50. Wyburn, K., et al. 2005. Macrophage-derived interleukin-18 in experimental renal allograft rejection. Nephrol. Dial. Transplant. 20:699-706.

51. Yin, J.L., et al. 2001. Real-time reverse transcriptasepolymerase chain reaction (RT-PCR) for measurement of cytokine and growth factor mRNA expression with fluorogenic probes or SYBR Green I. Immunol. Cell Biol. 79:213-221.

52. Wyburn, K., et al. 2006. Interleukin-18 affects local cytokine expression but does not impact on the development of kidney allograft rejection. Am.J. Transplant. 6:2612-2621. 